\title{
APORTES A LA FONETICA DIALECTAL DE SANABRIA Y DE SUS ZONAS COLINDANTES
}

\author{
CON TRISS MAPAS I,INGÜISTICOS
}

Bibliografia

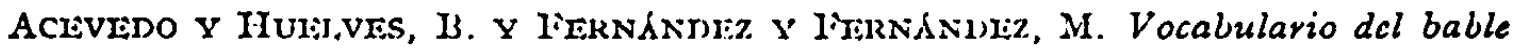
de occidente. Madrid, 1932.

Alonso, D. y Garcfa Yenra, V. I:l gallego-leonés de Ancares y su interés para la dialectologia portuguesa. CIEGall 196I, XI,VIII, 43-79.

AIVAR, M. Dialectologia española. Cuaderuos bibliogríficos, VII, 1962.

Arvardiz Bardón, C. De la Ribera del Orbigo. Cuentos de dialecto leonés s/1,1907.

Aracón y Fiscacrina. J, Entre brumas. Astorga, 1921.

Atlas Lingiïistico de la Penlnsula Ibbrica. Madrid, Ig62.

BLÁzULz l'raIld:, A. Limites del dialecto leonés occidental cn Alcañices, Puebla de Sanabria y La Bañeza. Menoria de la Junta para la Aupliación de Esstudios. Madrid, 1907, 67-78.

Canellada, M." J. El bable de Cabranes. Madrid, I944.

Casado Lomato, M.* C. El habla de la Cabreva Alta. Contribución al estudio del dialecto leonés. Madrid, 1948.

Castislanos, M. S. y otros. Limiles palatales en el Alto León, en TrDoRoLeon, I, 23-39.

CaTAY,AN MIinténdiz-PIDAI, D. El asturiano occidcntal. Examen sincrónico y explicación diacrónica de sus fronlevas cronólogicas. RoPh I956, X, 71-92; 1957, XI $120-158$.

Catarán Mioníndiz-Pidat, D. Resultados apico-palatales de -LL-, -NN- $y$ de LL- (<L-), NN- (<N-). RFE I954, XXXVIIT, I-44.

Catar.in, D. y Galmis, A. La diptongación en leonés. Archivum I954, IV, 87-147. Contribución lingiutstica del Magisterio, RDiTrPop, 196r, XVII, 525-560.

Contribuciones, cp. KRüCER, I.

Corrisiro, A. A lingua e a literalura pupular de Penamacor. Castclo Iranco, - I939.

Corominas, J. Vocabulario aranés. Barcclona, 1931. 
CORTES Y VAzQUEz, L. L. Dos textos dialectales de Rihonor y dos romances portugueses de Hermisende, en Misceldnea de Filologia, Literatura e História cultural, d memoria de Francisco Adolfo Coelho. II, Lisboa, 1950.

Cortes y VAzQUEx, L. L. La leyenda del Lago de Sanabria. RDiTrPop 1948, IV, 92-Ir4.

CoRTís $\times$ VגzQUEz, I.. L. El dialecto galaico-portugubs hablado en Lubidn (Zamora) Salamanca. 1954 .

Crespo Pozo, J. S. Contribución a un vocabulario castellano-gallego. Madrid, 1963.

Crews, C. M. Recherches sur le judéo-espagnol dans les pay's balkaniques. Paris, I935.

Cuesra, T. El protomártir asturiano Fr. Melchor Garcia Sampedro. Oviedo, 1895. Coverro PiNot. J. Diccionario gallego. Barcelona, 1876.

Dias, J. y hurculano di: Carvaluo, J. O falar de Rio de Onor. Coimbra, 1955. Dicc. enc. gall.-cast., cp. E. RODRíGUEz GONzAr.Ez.

Espinosa, A. M. Estudios sobre el español de Nuevo Mejico. I-II. Buenos Aires, 1930 y 1946.

Estanco Louro, M. F. DO ,O Livro de Alportel. Lisboa, 1929.

FarisIn, R. M. Nolas lingïlsticas sobre el habla de la Ribera del Orbigo en TrDoRoLeon, I, 4I-85.

Frrandindez, J. A. El habla de Sisterna. Madrid, 1960.

Alvarez Furnández-Cañedo. J. El habla y la cultura popular de Cabrales. Madrid, 1963.

Fernindez-GonzAlist, A. R. El habla y la cullubra popular de Oseja de Sajambre. Oviedo, 1959.

Fernándmz LIIERA, V. Gramatica y vocabulario del Fuero Juzgo. Madrid, 1929.

IERnandiz Muraliss, A. Ensayos poéticos en dialecto berciano. Leóm, 186x.

FIERRo, F. G. Algunas roces del vocabulario bable empleadas en Muros del Nalon. BIEstAst I952, VI, 24 1-250.

FINK, O. Studien über die Mundarlen der Sierra de Gata. Hamburg, 1929.

Garcia Blanco, M. Dialectalismos leoneses de un codice del Fuero Juzgo. Salamanca, 1927.

GaRCA DEL CASTILLO, J. y otros. Sobre el habla de la Cabreva Baia. TrDoRoLeon, I, 87-128.

Garcta DF, DIEGo, V. Elementos de gramática histórica gallega. Fonética-morfologia. Burgos, s/f.

Garch DE DIego, V. Manual de dialectologia española. Madrid, 1946.

GARCiA RIEY, V. Vocabulario del Bierzo. Madrid, 1934.

GARROTE, S. A. El dialecto vulgar leonés hablado en Maragateria y Tierra de Astorga. Madrid, 1947, 2.2 ed.

Goses Plerira, A. Tradiçies populares e linguagem de Villa Real, II, RL, 1909. XII 93-132.

Gra-RTendulizis, Li. Los muevos bablistas. Gijón, 1925.

Grossi, R. Aportacion al estudio del dialeclo de Campo de Caso. Archivum, 196r, III, 79-102.

Guzalin Alvartiz. El habla de Babia y Laciana. Madrid, I949. 
HaEnsci, G. Las hablas de la Alta Ribagorza (Pirineo aragonés). Zaragoza, 1960. HENRfQUEz UREÑ̃, P. El español en Méjico. Buenos Aires, I938.

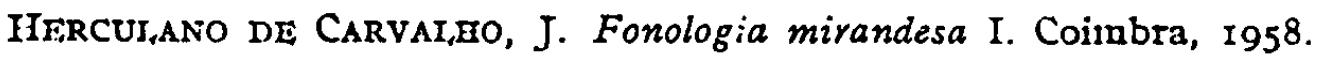

KRUGER, F. Contribuciones a la geografía léxica del NO. de la Peninsula. RDiTrPop 1957. XIII, 3-23.

KrUGrr, F. El dialecto de San Cipricin de Sanabria. Monografia leonesa. Madricl, 1923.

KrUger, I". Mezcla de dialectos. Homenaje a Menéndez Pidal, II. Madrid, I924. $12 \mathrm{I}-166$.

KRÜGER, F. Notas de dialectología asturiana comparada. BIEstAst I957, XI, $I-48$.

KRÜGER, F. Studien zur Lautgeschichte westspanischer Mundarten. Hamburg, 1914.

Lapesa, R. Asturiano y provenzal en el Fuero de Avilés. Salamanca, 1948.

IEITE DE: VASCONCFIIOS, J. Dialectos trasmontanos. RL II, 97-I20: III, 57-74.

LFITE DE VASCONCEI,J,OS, J. Esquisse d'une dialectologie portugaise. Paris-Lisboa, Igor.

IEITE dE VASCONCLir,os, J. Estudos de philologia mirandesa. I-II. Lisboa, r9ooroor.

LEITE DE VASCONCELLOS, J. Filologia barranquenha. I,ishoa, I955.

LEITE DE VASCONCII, I,OS, J. Linguagem de San Marlin de Trevejo (Cdceres: Hespanha) RL, I927, XXVI, I-16; XXXI, 167-246 (cp. Xalma).

LIEITE DE VASCONCIirLos, J. Opisculos, IV. Filologia, parte II. Coimbra, r929: I.inguagens fronteiriças de Hespanha e Portugal, 593-619; Linguas raianas do Trás-os-Montes, 723-738; Breve estudo dos falares de Riodonor e Guadramil, 739-790.

I,IITE DE VASCONCLi,IOS, J. Linguagens fronteirigas. Ermisende (ou Ermesende), Guadramil e Riodonor. RL I935, XXXIII, 307-309.

I EITr DE VASCONCEIr,os. Portugués dialectal da regiuo de Xalma (Hespanha). RL XXXI, I64-275 (parte I: San Martin de Trevejo, Ir: Eljas, III: Valverde del Fresno).

IENZ, R. El español en Chile. Buenos Aires, I940.

LINDLEY CinTRA, I. I. A linguagem dos Foros de Castelo Rodrigo. Lisboa, I959.

López, M. C. R. Aspectos del dialecto hablado en Aliste (Zamora), en TrDoRoLeon, I, I 29-I 42.

I.UGRIS FreIre, MI. Gramatica do idiona galcgo. A Cruĩa, r931.

Macundo, J. P. Dicionário etimológico da lingua portuguesa. I-II. Lisboa, 1956-1959.

MARTINS-SEQUEIRA, F. J. Apontamentos acerca do falar do Baixo Minho. Lisboa, $1957 / 58$.

Mentendez Garcis, M. Algunos limites dialectales en el occidente de Asturias. BIEstAst, 1951, n./ I4, 1-25.

Mantendiz Garcia, M. Cruce de dialectos en cl habla de Sisterna (Asturias). RDiTrPop, 1950, VI, 355-402.

Mrenrindiz Garcis, M. lil cuarto de los Valles. I. Oviedo, rog63.

MLnendez Prdal, R. Ll dialecto leonis, 2." cdiciún. Oviedo, 1962. 
MENENDEz PIDAL, R. Mamual de gramdtica historica española, II.", ed., Madrid, I962.

Mentendez Pidar, R. Origenes del español. Madrid, I950 $3 .^{\circ} \mathrm{ed}$.

Mezcla, cp. KRtrger, F.

MoksN, P. C. Vocabulario del Concejo de La Lomba en las montañas de Leon. $B R A E$, 1950, XXX, 313-330 y 439-456.

MuURa Santos, M." J. DE. Os falares fronteirifos de Trds-os-Montes. RPFil, 1962-1963, XII, 509-565.

MUNTHE, A. Anteckningar om Folkmalet $i$ en Trakt af Vestra Asturien. Upsala, 1887.

Netra Martinez, J. El habla de Lena. Oviedo, 1955.

Notas, cP. KRÜGER, F.

OenschiAger. A Medieval Spanish Word-List. Madison, I940.

ONfs, F. DE. Notas sobre el dialecto de San Martin de Trevejo. New York, Todd Memorial Volumes, r930-r931.

RATo y Hívia, A. Vocabulario de palabras $y$ frases bables que se hablaron antiguamente $y$ de las que hoy se hablan en el Principado de Asturias. Madrid, 1891.

Rodrfgutz-Casteri,ano, I. Aspectos del bable occidental. Oviedo, r954.

Rodriguez-CASTHiLANO, I. Contribucibn al vocabulario del bable occidenlal. Oviedo, 1957.

Rodríguiz-CAsTri,rano, L. El sonido s ( $<\mathrm{L}-,-\mathrm{I}, \mathrm{I}, \mathrm{g}$ ) del dialecto asturiano. Madrid. Estudios dedicados a Menéndez Pidal, 1953, IV, 201-238.

Rodrígez-Castiflano, I. La variedad dialectal del Alto Aller. Oviedo, 1952. RODRfGUEZ GONZÁIEZ, E. Diccionario enciclopédico gallego-castellano, I-III. Vigo, 1958-1961.

Rodrfguiz, MI. C. Aspectos del dialecto leonés hablado en Aliste (Zamora), en TrDoRoLeon, 1957, I, I 29-142.

Rubio Alvariz, F. Vocabulario dialectal del Valle Gordo (Léón). RDiTrPop, 1956, XII, 235-257; 1961, XVII, 264-320.

S. Cipridn, cp. KRüGIR, It.

SCINFIDER, H. Sludien zum Galizischen des Limiabeckens (Orense-Spanien). VKR, XI, 69-145; 193-281.

Thomas Pires, A. Vocabulúrio alemtejano. $R L, 1907, \mathrm{X}, 87-101$.

TORNer, I. M. Cancionero musical de la lirica popular asturiana. Madrid, 1920.

VIANA, A. Substulios para um vocabuldrio algarvio. Lisboa, 1954.

VIGón, B. Vocabulario dialectológico del Concejo de Colunga. 2." ed., Madrid, I955.

WAGNer, M. I. Caracleres gencrales del judeo-español de Oriente. Madrid, 1930. Westspanische Mundarten, cp. KRÜGER, F.

ZnMorA Vicenre, A. Dialectología española. Madrid, Ig6o.

Las abreviaturas de las revistas citadas en este articulo sun las siguientes: $A I L i=$ Anales del lustituto de Iingiilsticn. Mendoza. $A L P I=$ Atlas Lingülstico de la l'cuíusula Ibérica. Madrid, 1962. Archivum = Archivum. Oviedo. $B i D i H i=13 i b l i o t e c a$ de Dialcetologia Hispanonumericaua. Bucuos Aires. BIEstAst $=$ Boletín del Instituto de lisstudios $\Lambda$ sturianos. Oviedo. BRAE $=$ Boletín de 
la Real Acadenia Española. Madrid. CEGall = Cuadernos de Estudios Gallegos. Santiago de Conupostela. Interrogatorio $=\mathrm{cp}$. TrDoRoLeon. $N R F H=$ Nueva Revista de Iilologia Hispánica. México. RDiTrPop = Revista de Dialectologia y Tradiciones P'opulares. Madrid. $R F E=$ Revista de Filologia Española. Madrid. $R F_{0}=$ Romanische I'orschungen. Frankfurt am Main; $R L=$ Revista I usitana. Lisboa; $R P^{\prime} F i l=$ Revista Portuguesa de Filologia. Coimbra; RoPh $=$ Rounance Philology. Berkeley, Califoruia; TrDoRoLeon I, II $=$ Trabajos sobre el Dominio Ronuinico I,conés I, II. Madrid; $V K R=$ Volkstun und Kultur der Romanen. Hamburgo; $Z R P /=$ Zeitschrift für Romanische Philologie. Tübingen.

Con el presente trabajo continuamos una serie de estudios que estamos preparando sobre temas de dialectologia peninsular, particularmente del Noroeste. Publicamos al mismo tienpo, en Santiago de Chile, un artículo sobre Los alverbios "lejos" $y$ "luego" en perspectiva dialectal (con dos mapas lingiiisticos); ${ }^{1}$ estos y otros dedicados a temas fonéticos, morfológicos y lexicológicos serán reunidos en Muestras de un Atlas lingiïístico comentado de Sanabria y de sats zonas colindantes que pensamos editar en Madricl.

Iorman la base y el punto de partida materiales que recopilamos ya en el invierno de 192I-22 en Sanabria y en regiones vecinas (de León y Orense) y (que completamos en 1927 por encuestas realizadas en el extremo Sur-Oeste de la provincia de Oviedo (Asturias) y zonas rayanas de Galicia.

Constituyen nuestros estudios actuales, pues, una continuación y la fase final de los diversos trabajos que dedicamos a dichas regiones en años anteriores, desde la monografía El dialecto de San Ciprián de Sanabria del año I923 hasta el artículo El perfecto de los verbos -ar en los dialectos de Sanabria $y$ de sus zonas colindantes, publicado en el tomo XXXVIII, I954, de la RFE. En ese estudio ya anunciamos el proyecto que ahora está en vía de realización y "abrigamos", como entonces, "la esperanza de que el material recogido hace varios decenios tendrá aún hoy cierto valor». Por cierto, mientras tanto han aparecido, bajo los auspicios de las Universidades e Institutos de Mradrid, de Oviedo y de Salamanca, valiosas monografías y hasta obras sintéticas dedicadas a los dialectos de diversas regiones, desde Asturias hasta Salamanca y Extremadura, entre ellas también estudios que directamente afectan a zonas céntricas de nuestras propias exploraciones (sobre el asturiano occidental de I. Rodriguez-Castellano; sobre el Valle de R. Ibias, de nuestro inolvidable amigo Manuel Menéndez Carcía; sobre la Cabrera

1 Iin el Homenajc dedicado al Dr. Rodolfu Oroz, 1965. 
Alta, de M. ${ }^{2}$ C. I,obato, y sobre Lubián, en la periferia occidental de Sanabria, de L. L. Cortés y Vázquez). Nos complacemos en destacar, como ya lo hicimos en una ocasión anterior, el gran interés que tales y otras contribucioues a la dialectología occidental presentan para nuestros estudios de carácter comparativo; podremos seguramente decir algín día to mismo de una tesis doctoral de la Fiscuela de Coimbra de M. a J. de Moura Santos, Os falares fronteiricos de Trás-os-Montes, que trata problemas fronterizos desde el punto de vista portugués y de la que acaba de publicarse la Introducción (RP Fil, XII, 509 y sigs.).

Constituyen el punto de partida de nuestras encuestas e investigaciones Sanabria y sus zonas colindantes, región particularmente favorecida por la riqueza y variedad, el carácter profundamente arcaico y la vitalidad de sus dialectos. Por cierto, no faltan interpenctraciones castellanas, rinconcitos (del extremo Este) donde hasta ya se ha infiltrado el idioma oficial, pero predominan claramente aquellas zonas que, siguiendo una ruta multisecular, han conservado, hasta nuestros días, su carácter propio, mejor dicho los caracteres sumamente distintos que les ha imprimido la historia en el curso de los tiempos: el dialecto leonés en toda la zona central; el gallego, (puro, purísimo), netamente separado de aquélla (también geográficamente), en la parte occidental de la comarca, y en el extremo Sur, ya lindando con Trás-os-Montes de Portugal, una franja bastante extensa caracterizada por una mezcla curiosísinia de dialectos.

Es precisamente esa enorme variedad de aspectos la que nos induce a presentar nuestros materiales en forma de un Atlas lingüístico, excluyendo, en su mayor parte, lo que ya expusimos en ocasiones anteriores (vocabulario de la cultura material), pero incluyendo numerosos nuevos aspectos del léxico y de la fonética (poco de morfologia, ya que ésta se presta más bien a otro modo de exposición $y$, por lo tanto, la trataremos aparte). Para esclarecer bien el asunto agregamos que no fue uuestro plan, en el momento de la preparación y de la realización del viaje de estudios, editar algún día un Atlas o, por lo menos, Muestras de tal Atlas, si bien utilizábamos un cuestionario (más o menos completo y que fbamos completando en el mismo viaje). Este hecho explica que no todos nuestros mapas abarquen la totalidad de los lugares visitados (Sanabria y zonas colindantes, en total 67 , que corresponden a 5 del $A L P I)$; pueden aparccer pequeños vacios; pero, hasta en mapas como los de 'lejos' y de 'luego' que no queríamos suprimir, a pesar de ser incompletos, la exposición cartográfica presenta aspectos y problemas interesantes. $Y$ son precisamente estos los que constituyen el punto de partida de muestras exposiciones y discusiones, a las que daremos carácter comparativo - en el espacio, desde luego, y en el tiempo-, 
según lo exija el caso, en mayor o menor grado. Suponemos que, con esto, queda claramente circunscrito el plan y la finalidad de lo que llamamos Atlas lingüístico comentado de Sanabria. En fin: quisiéramos imprimir a materiales lingüísticos encajonados durante largos años nueva vicla - pues así lo merecen por su novedad- y dar un nuevo paso adclante hasta que se publique, algún día, el Atlas lingüístico de Sanabria en su forma completa, definitiva, programa al que el investigador salmantino $I_{\text {. }}$ I. $_{1}$ Cortés Vázquez ${ }^{1}$, mientras tanto, ha dado nuevos impulsos ${ }^{2}$.

\section{YERNO}

Interesan, sobre todo, el tratamicnto de la consonante inicial, de la vocal tónica y del grupo consonántico - NR-; observamos cierta variedad tambien en las formas del pronnmbre posesivo.

L, a consonante in icial presenta cuatro tipos distintos cuya difusión geográfica aparece netamente delimitada:

a) 'Todo el sector oriental de Sanabria ya dominado por la infiltración de la forma castellana (yérno, yérīno), fenómeno que hasta ha alcanzado partes de la Sanabria interior (S. Ciprián-Trefacio) y que

1 LuIS I. Contís y vizQuez. Solve el proyecto de realización del Allas lingüistico y etnográfico de Sanabria. Iin VII Congreso Internacional de Lingiaistica Románica. Universidad de Barcelona .1953. Barcelona. Abadia de San Cugat del Vallés. 1955. II. paigs. $635-6+5$.

2 I ugares cxplorados:

Zamora: r. Ribadelago; 2. San Martin de Castañeda; 3. Vigo; 4. Mrurias; 5. y 6. San Ciprián; 7. Coso; 8. Rábano; 9. Escudero; ro. San Justo; Ir. Barjacolo; 12. Porto; 13. Y'radorramisquedo; I4. Cepedelo; 15. Pias; 16. Villanueva; 17. Vinhais, Trás-os-Montes (Portugal); 18. Villavieja; 19. Hedradas; 20. Iubián; 21. Castrelos; 22. I,a lejera; 23. Hennisende; 24. Hedroso; 25. Aciberos; 26. Padornelo; 27. Pedralla; 28. Calabor; 29. Santa Cruz de $A$ branes; 3o. Rionor cie Castilla; 31 . Rionegrito; 32 . Sautiago de la Requejada; 33. Villar: 34. Onta; 35. Carbajales; 36. Veja del Castillo; 37. Villarejo; 38. Carbajalinos; 39. Doney; 40. Trefacio; 4I. Pedrazales; 42. Galcude; 43. Quintana; 4.. Limianos; 45. Sotillo; 46. San Román de Sanabria; 47. Cohreros; 48. Colomba de Sauabria; 49. Terroso.

León: 50. Quintanilla de Xuso; 51 . Valdavido; 52 . Truchas; 53. Iruela; 54. Iiucinedo; 55. La Baña; 56. Silván; 57. Lomba; 58 . Benuza; 59. Pombriego; 60. Castroquilame; 60*. P'ucnte de Domingo I'lúrez.

Orense: 62. Iardeira; 63. Casayo.

Leon (Bicrzo): 64*. I'ercje; 64. Trabadclo; 65. IHerrerias de Valcarce; 66. Iil Castro.

Lugo: 67 . Iil Cobrero. 
tiene su analogía en la zona colindaute, igualmente oriental de la provincia de León (50); yérno, según Casado Lobato, 54; observamos el tipo castellano $y$-también nuás hacia el Norte, en la misma provincia (Santa Lucia de Valdueza ${ }^{1}$; Lomba ${ }^{2}$; Oseja ${ }^{3}$ ) y en el sector colinclante de Oviedo (R. Ibias: Degaña: yénīo); yernu, Cudillero (conc. de Luarca), TrDoRoLeon, II, 32; como se ve, en todos estos casos igualmente en direçción Este *.

Opónense al tipo castellano toda una serie de formas que, en su repartición geográfica y evolución histórica, claramente revelan las tendencias evolutivas propias de las hablas occidentales.

Entre ellas merece, por nuestra parte, atención particular la etapa sonora

b) - por su carácter primitivo que, dentro del conjunto de los dialectos leoneses ${ }^{5}$, tan sólo se ha couservado en dos lugares fronterizos del extremo Sur de Sanabria: zénīo, Hermisende (23) y Calabor (28) ${ }^{6}$, en oposición a la variante sorda $\xi$-, tipica de amplias zonas colindantes de Zamora ?, León, del asturiauo occidental y hasta de zonas gallegas; ${ }^{8}$ pero en contacto directo con mirandés ž́nrru ${ }^{9}=$ port. genro (atestiguado desde el siglo X) ${ }^{10}$; lo misino en los dialectos portugueses de la Sierra de Gata, ž̨́yrıı ${ }^{11}$, de Alamedilla (Salamanca) ${ }^{12}$ y partes de la

1 TrDoRoLecn, I, 100: mio yenrro.

2 TrDoRoLeon, I, 94, 95, x16: yenro, mio yenrro; registrase la forma yienro, el miu yienro en el pueblo vecino La Baña; yo anoté, en r922, šípiño, lugar 55. como en las inmediacioncs; lo mismo sienrro, en Silván, según nuestras propias observaciones min sịniro, lugar 56.

3 A. R. ITERNANDEZ Gonzhirez. Oseja de Sajambre, 369: yenro; en cambio xineru 'enero', xabalin 'jaball', xclar 'helar' (ib. 50).

- Sobre variantes fonéticas del tipo castellano en la Sierra de Gata ýendru, etc., cp. FINK, 36, 78, frente a de Portugal), ib. 32, 33 .

- Cp. sobre la situación en general ZAMORA VICENTr, Dialectología española, 97. 99; FINK, 3 I y sigs.; SCHNEIDER, VKR, XI, 99 y sigs.; todos con referencias bibliográficas.

- Mezcla, 136 .

7 Mezcla, 136; S. Cipridn, 85 y sigs.

- Cp. infra s.-

- Herculano de Carvalino. Fonologia mirandesa, I, 35, 37, 49, iI7; sobre el tratamiento de J-en mirandés, LEITE DE VASCONCELLOS, Est. phil. mir. I, 269. 10 He aquí alguuas variautes dialectales de Portugal: jêrno, IEITE DE vASconcerulos. Filologia barranquenha, x80, al lado de jentlu 'gentio'; jêmê, jêmdda; janto, A. VisNA. Vocab. algarvio, 40.

11 ONís. San Marfin de Trevcjo, 64, 67; linisk, 32 con numerosos ejemplos del tratamieuto de J-.

12 IIiITE DE VASCONCIILOS. Linguagem portuguesa de Alamedilla. En Opuisculos del mismo autor, IV, 6r6, $\S$ I7: sobre $d j$ - africada sonora. 


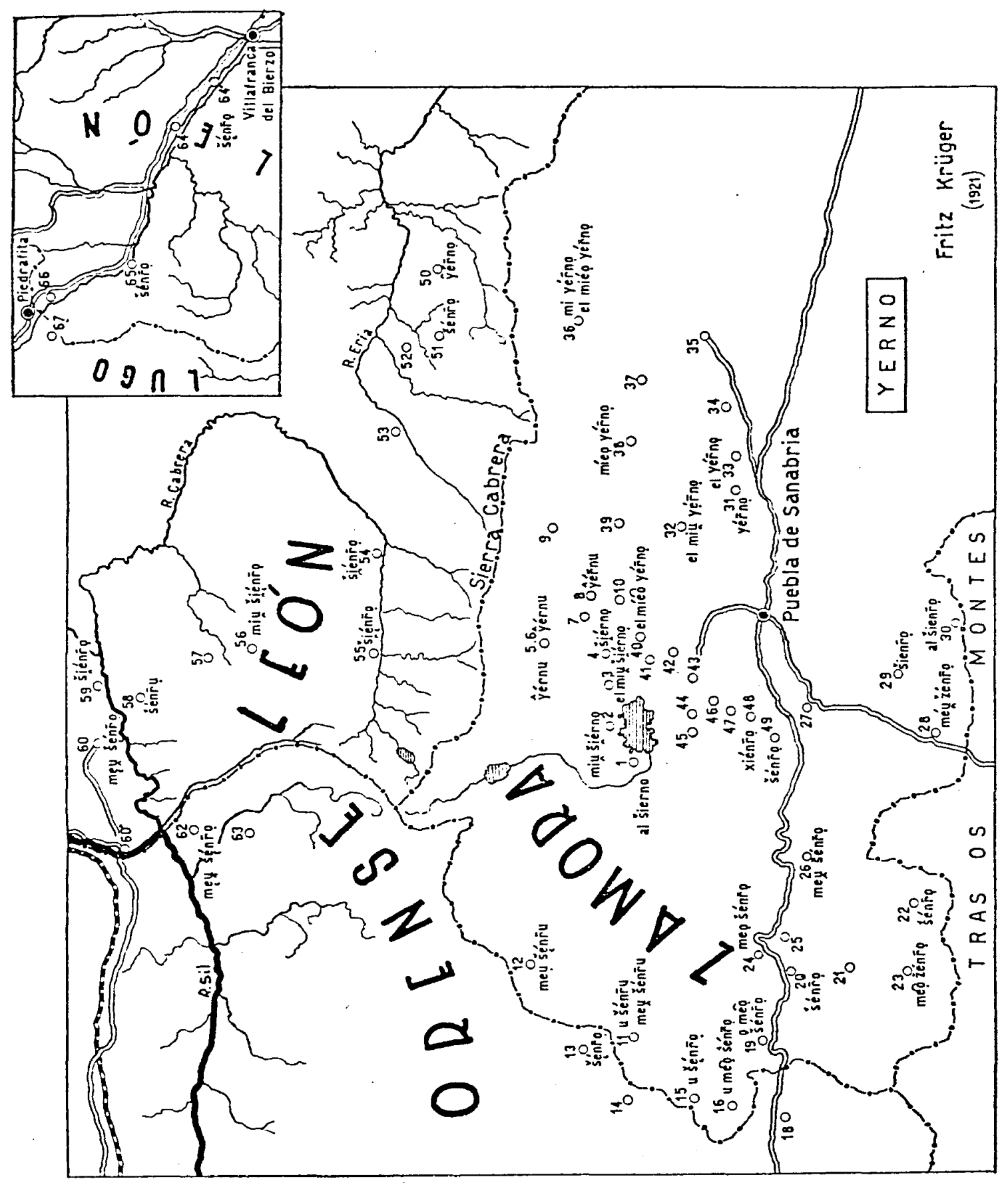


provincia gallega de Orense: žén $\ddot{r} u, \check{z} \dot{\tilde{e}}(n) \bar{r} u^{2}$, que igualmente han conservado el contacto con Portugal, vale decir la tradición anterior.

c) $\xi$ - La fricativa sorda $-s$, a la que ya nos referimos en el apartado b), represcnta, dentro de nuestro cuadro, la ctapa más ampliamente difundida, una etapa, a la vez, que alli - debiclo a una innovación bastante radical - debe haber sustituido la fricativa sonora conıún en tiempos anteriores. Como muestra claramente nuestro mapa, la fricativa sorda se ha mantenido - frente a la invasión castellana del tipo a)en una zona compacta, que abarca el centro de Sanabria (šierno, šiérno) ${ }^{2}$ partes del Sur (šienrịo, en rivalidad con žénirọ) y toda la zona gallega (šéñ̄o, - $u$, tipo común).

Hacia el Sur, la fricativa sorda se prolonga - pasando por Rionor de Castilla, 30: al šicn rọ- a Río de Onor (xienro) ${ }^{3}$ y Guadramil en 'Trás os Montes ${ }^{4}$, donde confronta claramente con el tipo sonoro propio del portugués ("gcuro, mirandés) ${ }^{5}$.

Hacia el Norte, la consonante sorda se continúa en la provincia de León, donde igualmente ocupa amplias zonas compactas: la Cabrera Alta y Baja (šiénrọ, 55, 54, 56, hasta Pombriego, 59), desde luego, el Bierzo (śénịo) y, más allá, hacia el Norte, el habla de Oseja ${ }^{\circ}$ de Sajambre (xente, xclada) ${ }^{7}$, Valle Gordo (šente, šelo), Babia-Laciana (šjermul) y Fornela (šiénro)-Ancares (š̨́ro).

Lo mismo puede decirse de Asturias, clonde, según A. Zamora Vicente, Dialectologia es.pañola, 99, "en la actualidad esta $\check{s}$ - inicial, —oriunda, de $\check{z}-$, es típica de todo el asturiano» ${ }^{8}$. Bastará mencionar algunos ejemplos de 'yerno': nosotros apuntamos en cl extremo SO de la provincia de Oviedo: šénīo, Besullo, Puerto de Leitariegos, Villar de Cendias

1 SCINIiIDIR. VKR, XI, IOO, 279. con otros ejemplos de $\check{z}$ -

2 S. Cipricin, 85-86.

3 Linte di: Vasconcelios. Opuisculos, IV, 750, 772, 790; Dras-Carvalmo. O falar de Rio de Onor, 6o; al lado de xente 'gente'; xelada 'hclada'.

- LiITE DE VASCONCEli,OS, Oprisculos, IV, 777, 789, 790: xiante 'gente'; xelar, xelada.

s Es notable la aparición de la fricativa sorda en el lugar fronterizo Valverde de la Sierra de Gata (š̉ıgru) frente a $\check{z}$-en los próximos pueblos de la misma zona

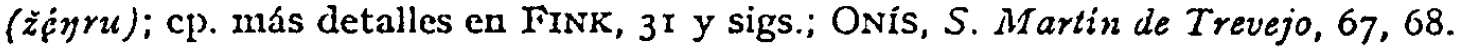

- íirnández Gonzáli:z, 368.

7 Iin Oscja aparcce yenro como castcllanismo (ib. 369).

- ZaMORA VICLNTE, 97: "en asturiano la fricativa medio-palatal sonora se ha ensordecido, probablemente desde el xvr, es decir desle la pérdida de la distinción entre $z$ sonora y sorda medicvales del castellanon; cp., sobre la $x$ asturiana, ya antes MisNENDiz PIDAI. El dialecto leonés $\S$ Io. 
(en el V. Río Ibias), al lado de yén̄ọo, Degaña; xicnru, Sisterna ${ }^{1} ;$ y encoutramos śćnro en el Cuarto de los Valles ${ }^{2}$, šénru, -0, en Villayón, Navelgas, Besullo ${ }^{3}$ (cp. nuestras propias observaciones) y otras partes del asturiano occidental (de Valdés al Lío) 4; la misma forma más hacia el lisste: xcuru, bable central ${ }^{5}$, Cabralcs ${ }^{6},-0$, Lena ${ }^{7}$; xcuru, sienru, Cabranes $^{8}$; xicuru, xenru, Colunga ".

Domina la fricativa $\xi$ - toda nuestra zona gallega: $\xi e n i r o, ~-u$, desde Castroquilame (60)- Lardeira (62) hasta Hedradas (I9) - Lubián (20) Hedroso (24) - Padornelo (26), incluso el Bierzo: Pereje (64) - Herrerías $(65)^{10}$, en contacto con las provincias gallegas, donde igualmente predomina xenro ${ }^{11}$.

Interesa que el proceso de ensordecimiento, al cual nos referimos antes y que, en muchos regiones, debe datar de tiempos bastante lejanos en algunos dialectos pueda observarse hoy día en plena evolución; así, eu diversos lugares del extremo Oeste de la Sierra de Gata (de habla portuguesa) ${ }^{12}$ y en la provincia de Orense ${ }^{13}$.

d) $x$ - quiere decir la jota española aparece como tipo aislado tan sólo en Colomba de Sanabria (48): xićnīo; corresponden a esta forma xéma 'yema', xielo 'hielo', xemjélgos 'mellizos'; xemindo 'gimiendo' en

1 J. A. I'ERNÁNDEZ $\S \S 9,35:$ xienru, xienti, xiema 'yema'.

2 M. Mrinéndez Garcí. El Cuarto de los Valles, I, 92: al sado de śénie, šildy, etc.

' R.-Castulinano. Aspectos, 125, 170: al lado de šente, šimélgos 'mellizos', etcétera; id., Contribucion, 424: xenvu general; sienru, Teberga.

- Zoun gallega; ACEVEDO, 227; $i b$. xente, xenelgo; ferro.

- R.-Casteli.ano. Alto Aller, 84, 107; R. Grossi. Aportacion al estudio del dialecto de Campo de Caso. Lin Archivum, XI, ror.

- Fernandez Cañedo, 214.

- NeIra, 3i.

- Cantelinda, 365.

- Braulio Vigón, al lado de xiente, xente; en un documento del año 1316 de Oviedo gienro.

10 Cp. también los datos contenidos en TrDoRoLeon, I, 100, I16, sobre la zona gallega de la Cabrera Baja: meu xenro, men $x^{\prime}$ enro, o xenro; meu senro (cp. infra); xenro, Puente de Domingo Flórez; xenrro, Castroquilame; xenro (FERNÁNDEZ Moraltes: Bierzo).

11 Los dicciouarios gallegos recientes escriben siempre xenro.

12 F. de Ovís. San Martin de Trevejo, 68, donde se registran zenti, senti; Euntus, suntus; Ecnrw; "puede observarse que la confusión entre $x$ y $j$ es grande $y$ que va gauando terreno la proumciación sordan.

13 Scnnlanmik, $\mid K l$, XI, 100: en ciertos lugares (Lovios, Eutrimo, etc.). predouvina $\approx-;$ pero ya aparece en cllos ocasionalmente la cousonaute homorgánica $\dot{s}$-; en otros pueblos se usa cxclusivamente esta última. 
ese mismo pueblo y la $x$ - en lugares vecinos del interior de Sanabria en casos como xicla, xjéla 'hieln' ,xiclo, xjélo 'hielo', etc.; xéma 'ycma'; xemiélgos, us- en 47, 5, 4, ximiélgos, 2, frente a šemićlgos, šimiélgos en el centro de Sanabria, Santa Cruz de Abrancs (29), šimélgos en la zona gallega $y$ żemćlgos en Hermisende $(23)^{1}$.

Corresponden estos ejemplos que, en el interior de Sanabria, ya se han arraigado bastante - confrontándose así con las etapas prinitivas preponderantes - a tendencias observadas esporádicamente en diversas zonas del lconés occilental 2: Oseja de Sajambre, jineru frente a xineru ${ }^{3}$; Astorga, jilada (halada, alada)'helada' ${ }^{4}$ frente la xilada en la Cabrera; Aliste, xelárc, téla, xanéírọ 5; Valverde (O. de Sicrra de Gata),

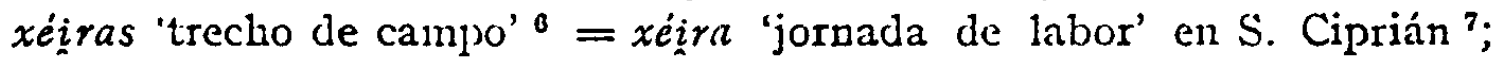
salmant. jera frente a źćira-šéira-šéra en otras comarcas del Oeste.

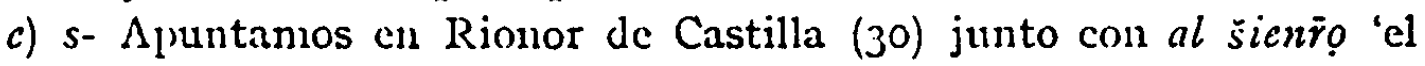
yerno' a siénte: 'la gente' con s- que aquí aparece esporidicamente, pero con toda seguridad. lincontramos luego, ya con mayor frecuencia, en la Cabrera Baja, sienrro, Silván, frente a xiela (TrDoRoLeon, I, 95. II6), según nuestros propios apuntes, miu siçniro, 56; $i b$., I27 xicnro; $i b .$, I26 sienro, xienro, xxienro, Castrillo de la Cabrera, $i b$. sugo, xugo, ensambre, enxambre (cp. además I, I00), también con la grafía xs: xsente (ib., I, 126, I27); men senro, Paradela de Nuces, en el sector gallego (ib., I, I0o) igual que sugo, xugo, x'ugo 'yugo de bueyes', sea, xea, x'cu matito 'hiela mucho' (ib., I, I0o); simelgos 'gemelos' Lardeira (lugar 62); aisladamente sineiru, junto a xineiru, Lena ( $T_{r}$ DoRoLeon, II, I43, I 42 ).

Opinamos que, en todos los casos citados, se trata de una variante fonética de $\check{s}$ - que no ha de ser absolutamente idéntica a s-.

I a vo o a l tó n i c a. Nuestro mapa indica claramente los diversos grupos dialectales que hay que distinguir en el tratamiento de la vocal tónica de GENERU:

a) la zona castellana, a la que ya nos referimos antes: yérno, -u en

1 Cp. sobre la situación de Sanabria S. Ciprian, 85 sgs.; Mezcla, r36-137: $z-s$. lisperamos poder completar este cuadro a base de muevos materiales.

2 Cp. también el resumen de ZaMORA VICENTE. Dial. esp., 99, con referencias a algunos casos asturiauos.

3 Iiernóndez Gonzíliiz. Oseja de Sajamure, 50.

- Garroti: 58 .

-Westspanische Mundarten, 241; TrDoRoLeun, I, I32, 137: yicla.

- Iink, 33.

7 Cp. S. Ciprian, 32. 
el Este y partes centrales de Samabria, 50, Quintanilla de Y.-I,eón.

b) la zona central de Samabria y la parte colindante de León, las dos de carácter leonés, arcaizante, en las que se conserva el diptongo

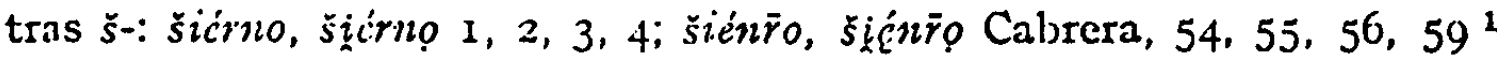
$y$ tras $x$-: $x i c i n \bar{r} 0$. 48 .

Eil misno feuómeno se continúa, jasando en el Sur de la provincia de Zamora por Santa Cruz de Abranes (29) y Rionor de Castilla (30): šieñ̄o, al extremo Norte de 'Trás os Montes, donde encontramos xienro en Río de Onor ${ }^{2}$ al lado de xiente 'gente' $(x=5)$ en el mismo lugar ${ }^{3}$ y xiente, en Guadramil 1.

Citamos a modo de comparación 'la gente':

29 šiéntẹ 23, 28 žéntẹ

30 siénte

El diptongo aparece tambiél más hacia el Norte en Fornela xićcıro

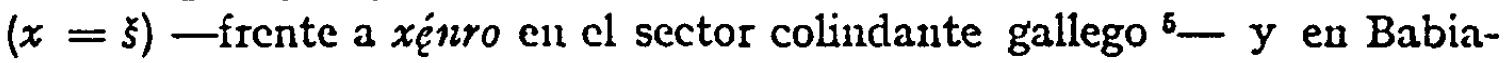
Laciann šjirnu, ${ }^{G}$ así como en ciertas comarcas asturianas: en el V. del Río Ibias xicuru ${ }^{7}$; 'leberga, sienru; Colunga, xienru-xenru, xientexentc 'gente'; Cabranes, $\zeta^{i} e n r u$, al lado de $x e n r r u{ }^{8}$.

1 Cp. sobre la Cabrera también los datos contenidos en TrDoRoLeon. I, 94, I19, 126, 127: mieu xienrro, Pombriego = nuestro lugar 59, miu xxienrro, Llamas de Cabrera, miu xienro, Trabazos, enxierno, xienro (ib. I, I27) Silván. según uuestros apuutes miu šiénìo. 56; cn cambio en el sertor gallego $i b$. I, 100: meu xenro, o xenro; CASADO LOBATO, 54, š'ema 'yema'.

2 LeITE dE VAsConcelitos. Opúsculos, IV, 772; DLAS-CARValHo. O falar de Rio de Onor, 6o; pero registrau estos últimos xente al lado de xienro. Véase sobre el carácter del diptongo la nota siguiente.

3 I.EITE DE V., IV, 750; cp. la nota anterior.

- IJEITE DI: V., IV, 777; véasc sobre el diptongo trauscrito ì id. IV, 734, 744, 745, 750: no idéntico al diptougo castellano, en Sendim, tierra se pronuncia casi como tirra; por lo tanto, cou acento en el primer elemento. Cp. sobre la prouunciación del diptongo más adelante.

- D. Alonso, Ancares, 46, 54 .

- Guzińn Alvarez, 330 igual que sjjénte 'gente'? etc.; hay que observar, sin embargo, que el elemento fricativo $j$ aparece también, y con toda regularidad, en casos cowo sjjára 'jarra', šjulebes 'jueves' etc.; véase nuestra nota en BIEstAst número 30 Notas de dialectología asturiana comparada: vocales tónicas, separata páginas 5-6: sonido de tránsito secundario. Morán, Lomba registra giente.

; J. A. FERNíndez § 17,67, con diptongación como en tienru 'tierno', § 35 xiema 'Yema', xienli 'gente'; xilar 'helar'. Nosotros apuntamos šnnīo en Besullo, Puerto de I.eitariegos y Villar de Cendias (este último en el sector gallego de dicho valle), igual que muita š́ntẹ eu Bes., mítja séntç en Villar, pero mujta šị̂ntẹ en Degaũa (yemo 'yierno').

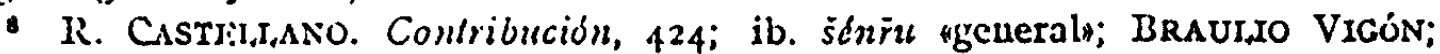
CANiLLAdDa, 365. 
c) las zonas gallegas de Sanabria y de León (Cabrera, 6o; Bierzo, 64, 65), sin diptongación: tipo šén rọ, $-\imath$ en contacto con Galicia $(13,62)$ y tipo žénrọ en el Sur de Sanabria $(23,28)$ en contacto con Portugal (genro).

Encontramos el tipo šénīo también en algunos lugares donde esperaríamos - según el tipo $b)^{1}$ - un diptongo: en Terroso $(49)^{2}$, Valdavido (5I) ${ }^{3}$ y 58 (Benuza) ${ }^{4}$. En todos estos casos nos encontramos en contacto inmediato con formas diptongadas; se tratará; pues, de una absorción secundaria o sea asimilación de los dos elementos palatales $(\xi i)$, proceso muy comprensible desde el punto de vista fonético.

Observamos el mismo fenómeno, más hacia el Norte, en tierras asturianas: en Besullo y Puerto de Leitariegos šén $\tilde{r} o$ al lado de xienrn, xienti 'gente', xicma 'y $\mathrm{sma}$ ', en Sisterna ${ }^{5}$ del mismo valle; en el Cuarto de los Valles "; Villayón- Navelgas - Besullo šcnru, -0 , al lado de šente 'gente' 7; y, más hacia el Este, Alto Aller, xcuro ${ }^{8}$; Campo de Caso, $x^{2 n r u}{ }^{\circ}$, Lena, xenro, Cabrales, šcnru; Colunga; xcnru junto a xienru, así como xente y xienlc; Cabranes, $\xi^{i} e n r u-x c n r u$, al lado de zente, xelu. Interesan estas últimas formas, ya que evideucian la cocxistencia de dos fases sucesivas de la evolución.

d) el grupo tónico -ie-. Contrariamente a lo que observamos en castellano, donde el primer elemento del grupo ie toma carácter semiconsonántico y más especialmente en el caso de yerno, donde la posición inicial de palabra conduce a un reforzamiento del carácter consonántico

I No insistimos en lugares donde se encuentra adiptongación a la gallega.

2 Fividencian el carácter leonés del lugar frontcrizo 49: fiel 'hiel'; kariéso 'mejilla'; mariélo 'amarillo'; pia 'pie'; abjéspura 'avispa'; ourẹ́llas 'orejas'; mál hacia el Norte con regularidad ié, ié (lugares 48 , etc.).

3 Indica también el tratamiento del grupo $-n^{\circ} r$ séńño, frente al castellanismo yérno, 5o, el contacto con las formas de la Cabrera: siénīo. Cp. sobre el contraste $-r n-\mathrm{y}-n \vec{r}-\mathrm{más}$ adelante.

- 58 Eù el mismo lugar ur đî́ntes, además, igual que en 59, siéla 'biela', miel, etc., vale decir con diptongación a la leonesa; en 60 ya nos encontramos en la zona gallega: męu šs̨niro.

- Cp. J. A. Fiernández $\S \S$ I7, 35.

- En el mismo lugar diptongación en piértiga, sienra 'conjunto de muchas hazas de terreno de labrantio'. La Tabierna topónimo, priesa 'prisa', etc. (MENÉNDEZ Garcí $\Lambda$. El Cuarto de los Valles, 24, 27).

7 R.-Casteriano. Aspectos, 125, 62: la $\varepsilon^{\prime}$ breve diptonga de un modo regular.

- Firnándiz-Cañeno, 33: Cabrales; R.-Castelinano. Alto Aller, 84, 107; 5 I vjespra 'avispa'; sjenda 'seudero'; rjésiro 'ristra'.

- Iin cl mismo dialccto: boticllu 'cstómago'; oriclla'; 'orilla'; escadiclla; fiel 'Licl'. Archivum, XI, ror. passim. 
del primer elemento - como atestigua claramente también la zona oriental de Sanabria y de León (mi yérno, el yérno, ŷernno, -u ${ }^{1}$-notamos, en la zona colindante de Sanabria así como en el Sur de León -las dos de carácter leonés - tuna mayor variedad. Eisto vale para el tratanicnto del grupo ic en gencral y el caso de yerno en especial, donde anotamos żiérno-şiéniro por una parte, con carácter semiconsonántico ${ }^{2}$ del primer elemento, y siénīo-xiénro y hasta šierno-sienrōo, con valor netamente vocálico, bisilábico de los dos elementos. Comprueban estos datos, que fácilmente podrian ser ampliados, en un caso muy especial, lo que ya queda expuesto en ocasiones anteriores sobre aspectos particulares de la diptongación de $\varepsilon$ en dialectos leoneses ${ }^{3}$ y trasmontanos ${ }^{4}$, particularidades que algún día tal vez merezcan un estudio sistemático y de conjunto.

Terminaremos las exposiciones anteriores con un ejemplo que evidencia un paralelismo perfecto en cuanto al tratamiento de la consonante inicial y de la vocal tónica E: *GEMELLICOS 'mellizos' cuya variedad fonética y terminológica nos revelará un mapa posterior:

żenćlgos, Hermisende y zona rayana portuguesa; $\xi c m i c l g u s-s c m e ́ l g o s$, šimélgos; xemiclgos, ximielgos.

- In-/-nI-El contraste establecido, desde hace siglos, entre GENERU castell. yerno IOI5 y port. genro 973 se manifiesta hoy día claramente en nuestros dialectos. Domina el tipo -rnn-, como es de esperar en la zona oriental de Sanabria, yérno, extendiéndose, en este caso hasta la región central alrededor del lago, donde aparece, con bastante frecuencia, en la forma siérno, šicrno. En todo el resto de la comarca se usa exclusivamente, a la manera gallego-portuguesa (xenro-genro), la forma contraria šćñọ, - $\imath \iota$, en todo el sector occidental, šénrọ, además, en 49 y xiénrịo en 48 , formas que ya discutimos antes; šienrōo, también

1 Is evidente cu csas comarcas la influencia castellana.

2 Corresponde uuestra transcripción en el terreno -que no vamos a modificar- a la $j$ semiconsonántica.

3 Cp. nuestra exposiciones en S. Ciprián, 21-25; Mezcla, 128-r29; los dos trabajos con mumerosos ejemplos de diversos tipos; MENENDEZ PLDAL, Manual" $\S$ Io"; CataldN-Galdies. La diplongación en leonés, 56 sgs;. Garrote, 90; Casado LoBATO, 37-38.

Sobre la situación distinta en el asturiano occidental cp. R-CASTELLANO, Aspectos, 62; Menéndez Garcia. El Cuarto de los Valles, 24, 25.

- Sobre el estado aún no bien esclarecido en los detalles cp. LEITE DE VASCONCELLos. Opísculos, IV, 734, 744, 745, 750; 774, 776 (Guadramil); w., Est. phil. mirand. I, § 50; Hinkcur,ano Carval,Ho, Fon. mir., I, 49; Mezcla § 7, 8: ie en Riovor de Castilla. 
en el extremo SE frente a žénīo hacia el SO $(28,23)$. La división geográfica resulta, pues, muy clara.

I.o mismo puede decirse de la provincia de Iseón con la única diferencia - diferencia muy notable- de que el tipo - $r n$ - queda limitado a la zona extrema oriental (en nuestro caso muy estrecha) y que, en todo el resto de la provincia, aparece, con homogeneidad absoluta, el tipo occidental -nr-: šén ro 5 I, šiénro, šiénīọ (54 y sigs.) ${ }^{2}$ en contacto con la zona gallega: šénīọ (60) ${ }^{2}$.

Quedan confirmadas nuestras observaciones en el Sur de la provincia de León (Cabrera Alta y Baja) por los clatos registrados en TrDoRoLeon, I 94,I I6 (sector leonés): el miu yienro (I a Baña), mieu (miul mienrro, mio yenrro (Lomba), sienrro (Silván), min xienro; I, 100 (sector gallego): meu xenro, meu senro, o xenro, mio yenrro (Sta. Lucia); en cambio xierno (ib. I. 96, II7, II 9 ).

Interesa destacar que el tipo -nr- se encuentra ocasionalmente en combinación con formas evidentemente castellanizadas como yienro, I,a Baña, mio yenrro, I,omba, mio ycurro, Santa Lucía de Valdueza; lo mismo yćn ro, en Degaña (R. Iljias), al lado de šéruro, Besullo, Villar de Cendias, pueblos vecinos; lo misno yenro, en el ayunt. de Oseja de Sajambre, provincia de León (Iiernández Conzález, 54).

Por otra parte el tipo -rn- (yerno) queda limitado -igual que en Quintanilla de Yuso (50) - también más hacia el Norte a la zona extrema oriental: xierno Castrillo de la Cabrera (TrDoRoLeon I, 96, II7), šjernu Babia-Laciana (Guzmán Alvarez 330) ${ }^{3}$ frente a xiéuro Fornela-xęnro Ancares en las immediaciones del NO de la provincia $(x=\check{s})$.

Volvemos al tipo -nr- en la provincia de Oviedo donde parece dominar amplias zonas: según nuestras observaciones, šén rọ, Villar de Cendias - Puerto de Leitaricgos- Besullo; xienru, Sisterna, en el mismo V. Río Ibias (J: A. Fernández $\S \S I 7,35,67)^{4}$; šenro, Cuarto de los Valles

1 Registramos en la Cabrera también bjénìes, 55, 56, $5^{8}$ igual que bínìes, 62, pueblo gallego vecino = vernes 'sesta feira' en el gallego del siglo xuv; bénies también en el sector gallego del $\mathrm{R}$. Ibias (Villar de Cendias); vienres en el bable occidental (R.-CASTELI,ANO. Aspectos 170; ID., Contribución, 450); en cambio biérnes 50, 54 (con $i$ vocal), bjērnes 60 , castellanismo.

Cabrera: tjérna, 52, 56, 58 -tjenra 55 = gall. tenro 'tiemo', tenreiro 'ternero', astur, occ. tinral, tenrala, vaca tinrera (R.-CASTri, LANO. Aspectos I70); etc.; GaRCía DE DIEGo, 6635 TENERU.

2 Sobre la posición de $5^{8}$ š́ninu en el límite de la zona leonesa cp. antes, nota.

- Sobre el elcmento $j$ cp. nuestras Nolas, 5-6; ZAMORA VICENTE. Dial. esp. 99; GARCfA DE DIYGo, 3072 simplifica xerno.

- En el misuo lugar tienru 'ticrno'; vienris 'viermes' (id.); cp). antes nota $\mathrm{r}$. 
(Menéndez García, I, 92); xenro, de Valdés al Eo, zona gallega (Acevedo, 227); šienru, Teberga ( $R$. Castellano, Contribución, 424) y, según el mismo autor (Aspectos, I70) šenru "lo normal es que se mantenga el grupo" en asturiano occ. ${ }^{1}$, de donde se prolonga al bable central: xenru, Campo de Caso ${ }^{2}$, senro, Lena $^{3} ; \xi^{i} e n r u, x e n r u$, Cabranes ${ }^{4}, x i e n r u$, xenru, Colunga ${ }^{5}$, sentru, Alto Aller ${ }^{6}$, senru, cnru, crnu. Cabrales ${ }^{7}$; encontramos como última estribación yenro en Oseja (prov. de León) ${ }^{8}$.

García de Diego, 3072 registra genro como ant. cast., yenro como cast. vulgar; andal. genro, $-a$ 'yerno y nuera' (Venceslada); genra = 'nora' también en Trás os Montes (RL, XII, IOI).

Todo el Oeste de la Península, incluso las zonas gallegas de Oviedo, León y Zamora, va dominado por el tipo $-n r-:$ gall. xenro -ženro, 9 port. genro.

Lo mismo žévyru, šévgru, d'éndru en el sector occidental de la Sierra de Gata (Fink, 33, 34). 'Tan sólo encontramos jêrno en el dialecto fronterizo de Barrancos ${ }^{10}$ y gérno, janro en Algarve ${ }^{11}$, probablemente en los dos casos por influencia española.

Particularidades de la cvolución fonética:

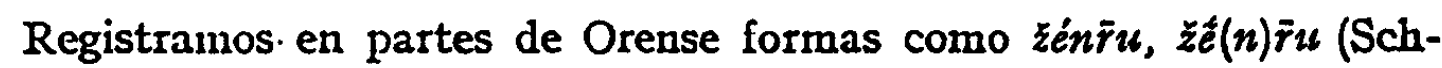
neider, VKR, XI, IOO) con nasalización de la vocal tónica; esta asimila: ción ha conducido, en mirandés, a una absorción completa de la consonante $n: \bar{z} \tilde{e}^{n} \bar{r} u, \tilde{z} \tilde{i} \bar{r} u$ (Herculano de Carvalho Fonologia mirandesa, I, $35,37,49)$.

En la evolución del grupo $N^{\prime} R$ ha sido destacado ${ }^{12}$ el reforzamiento de la articulación de la $r$ "que se hace vibrante múltiple» en asturiano y

2 Cp. antes nota I de la p. 265: vienres, tinral; MENEndez Garcia. Limites, 20, 21: venres, tenro, senra 'terreno labrantio, dividido en muchas hazas'; D. CATALAN. RoPh, X, S7.

s R. Grossi, ior.

3 Neira, 3 I.

- Caneliada, 365; ib. tienru, pág. 350.

s BR. VIGós, ib. tienru.

- R.-Casteliano. Allo Aller 107; ib. tienru, pero terral.

7 a. Fernández Cañedo, 214.

- Fernández GonzÁler, 54: yenro, tienro.

- Schneider, IKKR, XI, too; CuVEIro PiÑol. Dicc. gallego, 148, registra genro \& aut. gerno.

10 IEITE DE VASCONCELLOS. Filologia barranquenha, 180.

II A. VInNa. Subsidios para um vocabulario algarvio, 42, 46; EstaNco LoURO.

O Livro de Alportel, II, 243 registra gerno = 'género'.

12 R.-Castelinano. Aspectos, i7o; Meníndez Pidal. Manualu $559^{4}$. 
otros dialectos. De ello da una prueba perfecta nuestro mapa, en el que aparece con frecuencia $-\bar{r} n-\mathrm{y}$, con toda regularidad, $-n \bar{r}-$.

"Phonèmes additionnels" de carácter curioso en diversas regiones

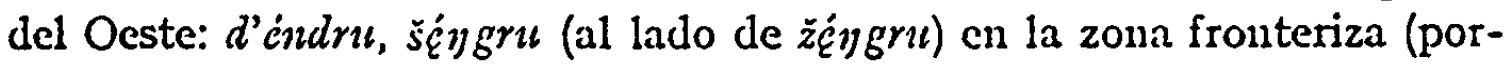
tuguesa) de la Sierra de Gata (Fink, 32, 33).

genlro, Bragança (RL III, 70), forma que puede ser comparada con telro 'tierno', olra 'honra' en dialectos extremeños (Leite de Vasconcellos. Est. phil. mir. I, 282); frente a tienro, en Miranda y Río de Onor, ib.; id., Opriscrelos, IV, 75I.

genlro, Alentejo (RL, X, 90, 245).

gêlro 'genro', têlro 'tierno' Beira (Cordle'ro, Penamacor, 79).

Particularidades morfológicas:

Artículo definido. De entre las formas del artículo masculino ${ }^{1}$ llama la atención la coincidencia obscrvada cntre al šicrno, Ribadelago, I, y al sienrọo, Rionor, 30, dos pueblos que, si bien distantes, reflejan en este y otros respectos, dentro del dominio leonés, una tradición arcaizante de mayor interés. Explicamos en Mezcla $^{2}$, I43, la forma al por la tendencia a oscurecer el timbre de la partícula proclítica, fenómeno observado también en el Norte de Portugal ${ }^{3}$.

Pronombre posesivo. La forma masculina del singular anotada en algunos lugares de nuestro mapa representa cierta variedad resumida en el esquema siguiente ${ }^{4}$ y que podria ser ilustrada por numerosos otros ejemplos:

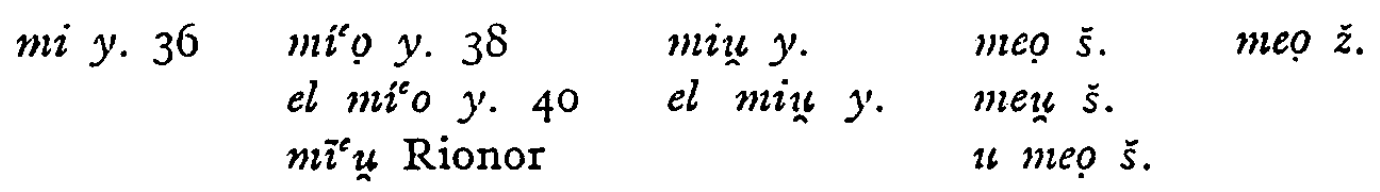

Corresponden las variantes dialectales - la forma castellana mi es muy rara - por una parte al tipo leonés-trasmontano: mío, miu, por otra al tipo gallego: meo, meu ${ }^{5}$.

1 Cp. S. Ciprián, 94-95.

2 Cp. ib. las formas $e l, 0, u$ en el Sur de Sanabria.

3 ITITE dE VASCONCELI,OS. Est. phil.mir., I, 357-358.

- Corresponde $y$ a yerno, $\dot{s}$ a šenro, ż a żenro.

- Cp. S. Ciprián, 93; Mezcla § 38. Son notables las congruencias entre las variantes recopiladas por nosotros (en 192I) y las registradas en la literatura regional ("Entre brumas", etc.), y por párrocos de la Cabrera en interrogatorios escritos confeccionados treinta años después; cp. por ahora TrDoRoLeon, I, 94, 100:

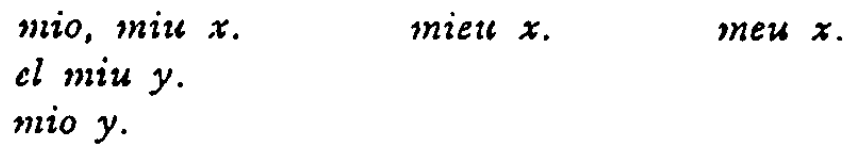




\section{LAS (DOS) OREJAS}

El mapa oreja presenta dos aspectos principales: el tratamiento de la sllaba inicial y la variedad de los reflejos del grupo consonántico -C'L.

L, a $\mathrm{s}$ il a b a in i cia 1 . Según tendencias frecuentemente observadas en las hablas occidentales, incluso el gallego y el portugués, la v o c a 1 i n i ci a 1 de ORICLA tiende a convertirse - como o-protónica también ell otras condiciones- ${ }^{1}$ en una 0 - bastante cerrada y hasta en la vocal extrema $u$-. Esste fenómeno, del que nuestro mapa suministra una idea muy clara (empleo casi exclusivo de $o$ - y $u$, en el caso de no diptongarse la vocal protónica), puede ser ilustrado, además, por casos como oveja, orilla, etc., en los cuales encontramos exactamente los mismos resultados: gall. obẹlala, utẹlas, leon. ubélas, obéya, obéxa, utéxa, etc.;

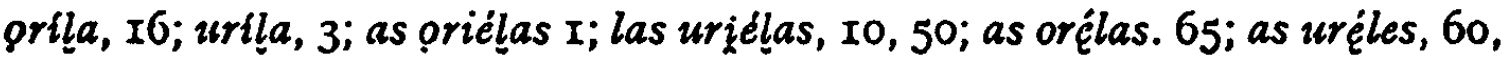
62; urileíro top. de un monte 40 , etc.

Pero observamos, además, otra modificación que afecta a la misma vocal. Trátase de la diptongación de la o-protónica en posición inicial de sílaba. Lo que da a tal fenómeno una nota particular es que, dentro del margen de nuestras hablas, queda estrictamente limitado a los dialectos leoneses ${ }^{2}$, donde alterna, pero con preponderancia absoluta, con la tendencia de cerrar la abertura de la misma vocal, según vimos en el párrafo anterior. Así, predominan en la Cabrera dries ouréyas, dues auréyas y en la zona céntrica de Sanabria y hacia el Sur (cp. 29, 30) ducs puréilas, ouréyas, as aurélas (29), dus auréyas (30) y hasta auréxa; lo mismo apuntamos 'las orillas de una corriente de agua': as aunriélas, $l$ aurriéla en la Cabrera y en Sanabria as qurílas (del río- 45), ourílas rièu (32), ouriéla 'límite del campo, del monte '(5), auriélo 'banda de lana que las mujeres colocan a modo de faja' (38), $l$ aurilia (del río) (8), etc. Son particularmente frecuentes y, en ciertas regiones de uso casi exclusivo, las variantes de 'oveja', problamente debido al influjo labializante de la -b-siguiente: así, en la Cabrera,

1 He aquil algunas refereucias: Westspanische Mucndarten \$§ 149-159; FunK § 31; S. Cipriän § 34; Mezcla § I3; R.-Castel cano. Aspectos § 32, etc.

2 Cp. sobre la difusión geográfica del fenómeno nuestro artículo Los adverbios 'lcjos' $y$ 'luego' en perspectiva dialectal: o- ou- en posición inicial absoluta, con referencins bibliograficas y una scrie de ejemplos (ougano 'hogaño', oujuallá 'ojalá', ourilla 'orilla del rio'.) 
Qúbéya, las outóyas; en el centro de Sanabria, outbéla, oǔbéilla, oútéxa; etc.; cp. TrDoRoLeon, I, 30, 94.

Desde León el mismo fenómeno se prolonga a Asturias ${ }^{1}$, particularmente al bable occidental y, en el Sur, a algunos puntos del extremo Norte de Portugal (Miranda, Río de Onor) ${ }^{2}$ y a la Aliste ${ }^{3}$.

Muestra el ejemplo (aislado) aucrélas 'orillas', registrado en el pueblo gallego r4, Cepedelo, que la evolución descrita en los párrafos anteriores fue favorecida y en ciertos casos - como el presente de la zona gallega- hasta iniciada por el empleo de la palabra con el artículo femenino (a uréla-ıına uréla) y la falsa coordinación de los elementos iniciales, como evidencian grafías que encontramos en el sector gallego de la Cabrera Baja en el caso de 'con la oveja': co a ovella, cu a ovella, qua obella, caubella, con auvella (TrDoRoLeon, I, roo).

Evidencia, en cambio, nuestro mapa que formas diptongadas faltan completamente en las hablas gallegas vecinas: en el Bierzo, 64, orélas, en la zona gallega del $\mathrm{R}$. Cabrera, 60, en las provincias de Orense y en los scctores gallegos de Sanabria (I2, II, I5, I6, I9, 20, etc.). Encontramos, sin embargo, en Crespo Pozo 'oreja' gall. orella, en algunas partes ourella; 'orilla' orela; Crónica Troyana, ourela.

-C'L-, -KL- Por instructivo que sea el tratamiento de la vocal protónica de AURICULA, ORICLA puesto que ya nos revela una imponente variedad regional, no interesa menos la evolución del grupo consonántico -C' $L_{-}-$, $-\mathrm{KL}$-, cuya diversidad, en un dominio relativamente limitado, nos permite precisar los datos alegados por otros autores a ese aspecto característico del leonés ${ }^{4}$.

Distinguimos, dentro del área estudiada por nosotros, cuatro grupos, caracterizados, en su mayoría, por una marcada uniformidad, la que, a su vez, revela etapas arcaicas de gran interés.

No pueden faltar formas castellanizadas; pero estas aparecen en Sanabria - contrariamente a lo observado ell otros casos (como p. ej. 'ojo') - tan sólo esporádicamente, sobre todo a lo largo de

1 Citaremos a modo de ilustración provisoria del extremo SO de Oviedo dos ejemplos típicos: las "orejas" del arado: oréas, Pambley; uréas, Besullo; ouriciéíras. Degaña, Tablado, Bao; outuiléiras, Villar de Ce. (Ibias), utriléîras, Folgoso, Gancio (estos últimos situados en zona gallega); "orejas" del timón del carro que sirven para atar el yugo: oréas, Trones; uréas, Besullo; oureca, Puerto de Leitariegos; orexas, Pambley.

2 Véanse referencias cu el artículo citado en la nota 2 de la p. 268.

3 TrDolioLeon, I, 94, I34, 135, I40: onveya, oufrenda, ouraciones.

- Cp. recientemente el resumen de Zamora Viceñe. Dial. esp., ro6-107. 
la gran carretera, escaseando ya en el interior y faltando completamente en el Oeste y Sur de la comarca, desde luego también en la zona gallega.

El proceso de la infiltración de elementos castellanos en nuestra comarca - tan variado como complicado- merecería algún día un estudio más detenido. Mencionaremos en cuanto a nuestro caso especial -como mera muestra- los aspectos siguientes:

a) subsiste, al lado del préstamo moderno, la forma arcaica en la memoria del pueblo: auréxa-ouréles 5, 6; uréxas-uréyas, 35.

b) la forma castellana vale como designación de la parte del cuerpo humano; la otra, dialectal, para la oreja del cerdo: oréxas-ourellas, 3.

c) se hace una distinción parecida entre las uréxas del hombre y las uriyéíras del arado en el pueblo 33.

d) generalmente la forma castellana se modifica según las tendencias dialectales de la pronunciación: ouréxes 5 (cambio de -as en -es); uréxas, Io, $33,35,43,46$ (con el cambio bien conocido de la vocal protónica); alucicia, 5 .

e) y se combina con clementos tradicionales de la morfología, como p. cj. ducs oréxas, 32 'dos orcjas'; artículo as 'las', en otros casos.

$f$ ) hay pueblos en la zona oriental de Sanabria (donde hay que presumir una interpenetración castellana considerable) que, al lado del préstamo oreja, conservan numerosos rasgos tradicionales del grupo $K^{\prime} L^{1} ; 32$, dues oréxas, también abéxa 'abeja', abexón, pero tagáyo de xénte 'porción de gente' -en otros lugares (I, 3, 6, 8, 44, 48) tagálo, $-u=$ gall. tagálo, trasmont. tagalho-; cukáyọ 'cencerro' = ćokáyo, 39 , ćukálo, -u, 5, ro, 45; un agriya 'aguja', igual que en 36, 38, 39 y agúla en otros lugares ${ }^{2}$.

Opónense a las imnovaciones mencionadas en el párrafo anterior las tendencias a rcaizantes que dan a la mayor parte de Sanabria y de la provincia de León un carácter particular. En ellas las etapas primitivas de la evolución de - KL - (- $\mathrm{L}_{\text {- }}$-etc.), etapas que, en parte, remontan a la Edad media más lejana, se han conservado en zonas compactas con una perfección admirable, contrastando así totalmente con la evolución castellana. Nos referimos a las etapas $-l-$ y $-y-$, cuya difusión geográfica vamos a trazar en pocas líneas a base del ejemplo 'oreja'.

I a - $l l-(-l-)$ g a 11 e g a ocupa, en contacto directo con Orense (nuestros lugares I3, I4, 62), la zona rayana occidental de Zamora (de Porto

1 Cp. también nuestras exposiciones en S. Cipridn, 83-85.

2 Mapas futuros de 'abeja', 'aguja', 'ojo', 'hoja' etc., nos darán otros tantos puntos de partida para una discusión más detenida. 


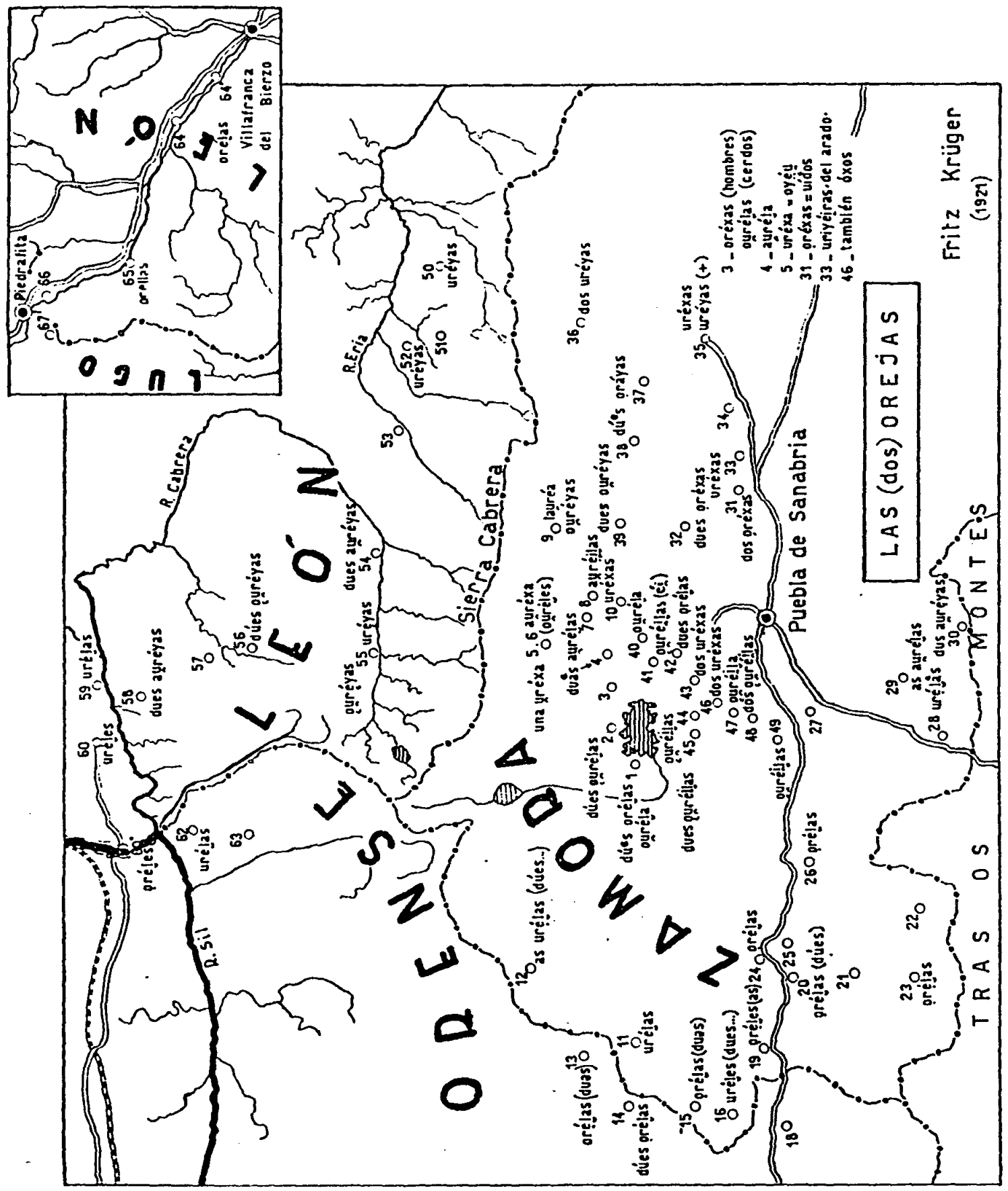


I2 hacia el Sur I9, 20, 24, 26) ${ }^{1}$, los lugares fronterizos 60 y $60^{*}$ de la Cabrera Baja (León) ${ }^{2}$, el Alto Bierzo (oréla el Oeste), y 11 rinconcito del extremo NO del Alto León ${ }^{3}$ (incluso Ancares) 4; todas ellas, zonas de habla netamente gallega ${ }^{5}$.

I a $-l-1$ c o n e s a. Registramos en esta rúbrica las zonas y los lugares de provincias leonesas que - sin tener carácter gallego, pero sí vecinas a las mencionadas en el apartado anterior- conservan el reflejo - $l$ - como rasgo fundamental, como residuo antiquísimo y hoy día rarísimo dentro del amplio dominio leonés. Nos referimos, en primer lugar, a aquella zona céntrica de Sanabria que, en forma de franja larga y bastante extensa, va dominada por el tipo oréla, ourréla, ouréila a auréila, desde San Ciprián y los alrededores del pintoresco lago (I, 2, 40, 4I, 42, 44, 45) hasta Cobreros (47), Colomba (48) y Terroso (49), lugares ya situados en las inmediaciones de la gran carretera, de donde dicha zona lingüística se prolonga directamente a la faja periférica del Sur, que, en su mayor parte, ha conservado exactamente el mismo tipo: desde Hermisende (23) orẹ́las-urę̧la le Calabor (28) hasta Santa Cruz de A. (29) as aurélas".

Problemas locales. Presenta uno de los problemas más atractivos de esa categoría el pequeño triángulo formado, en el sector extremo occidental de la Cabrera Baja, por los lugares Benuza (58), Pombriego (59) y Castroquilame (60). Si el dialecto de Benuza puede ser considerado como esencialmente leonés y el de Castroquilame como netamente gallego, el habla de Pombriego se encuentra, entre esos dos polos, como estación de cruce entre dos mundos distintos. Sin agotar la materia, a cuya discusión da lugar precisamente el tratamiento del grupo - KL- en el caso 'oreja' (y sus similares), registraremos, en forma esquemática, algunos ejemplos cuyo carácter evidencia, a primera vista,

1 Véase sobre la zona meridional, igualmente $-\underline{l}-$, mús adelante: $-\underline{-}$ - leonesa.

2 Cp. también los datos registrados en el Interrogatorio publicado en TrDoRoLeon, I, solvre el sector gallego de dicha comarca.

3 TrDoRoLeon, I, 30, 33: orella, ovella; folla 'hoja', muller 'mujer' en Villarbón; en cambio según GARCía REY: oreyón 'orejón', Folgoso de la Ribera; oveya, Molinaseca.

1 D. Alonso. Ancares, 58.

3 Cp. sobre la zona gallega de Asturias más adelante.

- Pueden verse más ejemplos de la conservación de la autigua -l- en $S$. Ciprian $\S 61$ y Mezcla $\S 33$.

Cp. sobre el tratamiento de -KLr en los dialectos leoneses $\left(-b_{-},-y-\right.$, etc.), últimamente Zamora ViCENTE. Dial. esp., 106 y sigs.; D. CATAIAN. El asturiano occidental. RoPh, XI, 130 y sigs. 139 . 
el interés que ofrece el tema de mezcla de dialectos - que ya hablamos discutido con motivo de otra zona también periférica del Oeste- en aquel rinconcito solitario de nuestra montaña.

Iriguran dichos lugares en los Interrogatorios escritos publicados en TrDoRoLeón I, 92 y sigs., II6 y sigs., โ25.

\begin{tabular}{|c|c|c|}
\hline $\begin{array}{l}\text { 'orejas': } \\
\quad \text { auréyas, } 58\end{array}$ & urélas, 59 & uréles, 60 \\
\hline $\begin{array}{l}\text { 'ovejas': } \\
\quad \text { as aubbéyas, } 58\end{array}$ & ubélas, 59 & ubéles, $\epsilon$ \\
\hline $\begin{array}{l}\text { bejas': } \\
\text { abéyas, } 58\end{array}$ & abélas, 59 & abéles, \\
\hline
\end{tabular}

Observamos tal distinción entre la - $y$ - leonesa (58), la - $l$ - gallega (60) y la - $l$ - de 59 , lugar que forma parte del leonés, en otros numerosos ejemplos, incluso en la toponimia local:

58, uombres de montes: tayadẹ́̂rq; labiyóna, este último relacionado con los ejemplos siguientes, derivados de 'abeja', 'abejón':

la biyéira, monte de $39=(a)$ biera 'colmenar' (Morán, Lomba r3); abeliera 'colmena' Zamora occ. (Zamora Vicente. Dial.esp., I08), Abejera, nombre de lugar, Zamora (Nomenclátor), topónimo frecuentísimo en tierras gallegas, desde luego con -ll-: a purtéla d abiléira, Porto, I2, Penalvelleira La Coruña, Abelleira (de Abajo, Arriba) Orense, La Coruña, Lugo (al lado de $A$ belleiroá), A bellal Orense, $A$ bellás Orense, $A$ bellas L,a Coruña, Abellariza (ib.). ${ }^{1}$ yerta biyęíira, 55; biléîra, 59; abiléire, 60, planta medicinal como gall. herba abelleira, abilleira (Crespo Pozo, 424).

'noche':

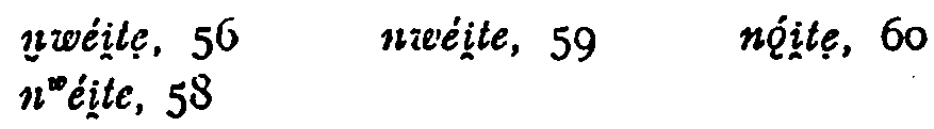

'ojos':

$\begin{array}{ll}\text { wéyos, } 58 \quad \text { óxos, } 59 & \text { éxọ, Q́lọ, } 60 \\ & \text { élọ, 60* } 62\end{array}$

'hoy':

\begin{tabular}{|c|c|}
\hline $\begin{array}{ll}\text { gwęi, } & 56 \\
\text { gwei. } & 58\end{array}$ & $\begin{array}{l}\text { Qí. } 59 \\
\text { guey, } 59\end{array}$ \\
\hline
\end{tabular}

1 Los nombres de lugares citados según el Nomencldtor. 
En el ejemplo 'noche' Pombriego (59) participa claramente en la diptongación característica leonesa de la vocal tónica ante palatal ${ }^{1}$. El misno diptongo ha sido registrado alli en el Interrogatorio de I953-54 en el caso de guey 'hoy' (al lado de ói $i$ cp. más abajo) y en: you cuello 'yo cojo', me mucllo 'me mojo' 2; freélas (citado en S. Ciprián, 27, nota $I$, ejemplos análogos del Sur de León) ${ }^{3}$.

En el caso de 'ojo(s)' aparece el castellanismo óxo (59) bastante frecuente en otras zonas leonesas y que hasta empieza a propagarse en el pueblo vecino gallego (60).

En cuanto a o $i$ 'hoy' (59), se trata también de una forma importada igual que en el rinconcito vecino de habla gallega $\left(60,60^{*}\right)$, en contraposición al gallego auténtico hoxe, huxe $(x=\check{s})$, incluso óse en la zona gallega de Sanabria; representa la forma auténtica de 59: guey (cp. más arriba).

El límite observado en nuestro triángulo entre la vocal diptongada (nwéîte, wéyos, gwéi-guey) ${ }^{5}$ y la adiptongación típicamente gallega (nóite, ólo, ošc 'hoy') se prolonga en forma muy marcada tanto hacia el Sur (hasta la zona meridional de Sanabria ${ }^{6}$ ) y más allá a 'Trás os Montes ${ }^{7}$ y la Aliste ${ }^{8}$, y hacia el Norte (Alto Lcón ${ }^{9}$-liornela-Ancares ${ }^{10}$ - V. del Río Ibias, ${ }^{11}$, etc.) ${ }^{12}$.

1 Cp. en el capitulo siguiente: interpretación del mapa 'noche'.

2 TrDoRoLebn, I, 92.

3 Cp. sobre la Cabrera Alta, CaSado Lobato $\$ 4$ : fweya etc.; TrDoRoLeon I, 30, 92,97 .

- Registramos con satisfacción la concordancia con el Interrogatorio escrito del año $1953 / 54$ donde Pombriego igualmente figura con el castellanismo ojo ( $T$ rDoRoLeon, I, 92), frente a ueyos en otros lugares leoneses vecinos.

- Véase el resumen de ese aspecto en ZaMora Vicrente. Dial. esp., 75-76 y Catalín-Gaimmes. Diptongación, 26 sgs., 30 sgs., 40 sgs.; materiales recientes en monografías locales; $c p$. algunas referencias en las notas siguientes y observaciones sobre detalles del problema en el cap. dedicado al mapa 'noche' (parte final del articulo presente).

- S. Ciprián § I2; Mezcla §9.

'Lisire, Opitsculos, IV, 746 uoyo 'ojo', ib. 775; IV, 75I uoiyo 'ojo', nuoite 'noclee'; IV, 764 buoi, 768 nuoile, 765 cuoiro; IV, 774 uôio 'ojo', Guadramil; TrDo RoLeon. I, 133 güeyos, Guadramil ;DIAS-CARVALuo, Rio de Onor 17, 18; LEITE. Esq. I99

- Westspanische Mundarten §§ 100, 103; TrDoRoLeon, I, 133, 134: mueyo 'mojo'.

- TrDoRoLeon, I, 30: fuella, fueya, fuecha = folla 'hoja'.

10 D. Aronso. Ancares, 46, 53: nueite, guei, guello, Fornela-frente a ollo, etc. Ancares.

11 R.-Castelifano. Aspectos, 73; nuestras Notas; Mentendez Garcfa. Cruce, 360; id. Limites, I7. 18; Sisterna.

12 Cp. también el resumen de Zasors Vicente. Dial. esp., 75-76. 
Interesa, por otra parte, la delimitación de la nasal palatalizada in- común a la zona meridional de la Cabrera (nwéíle, $-e, 55,54,56$, inueite, Sigüeya) ${ }^{1}$ frente a nueite, Lomba, según TrDoRoLeon, I, 92, II6; = lugar $57, u^{u}$ éítc, $58=n w e ́ i t e, 59$, lugares leoneses en cuanto a la diptongación (-réi-), pero vinculados con el habla gallega (nóite, 60, 63; nóutc, 62) en cuanto a la $n$ - inicial no palatalizada. El caso resulta un poco complicado, ya que también en otras regiones leonesas "cada día van introduciéndose más formas modernas ( $\operatorname{con} n$ - en lugar de $\tilde{n}) n^{2}$.

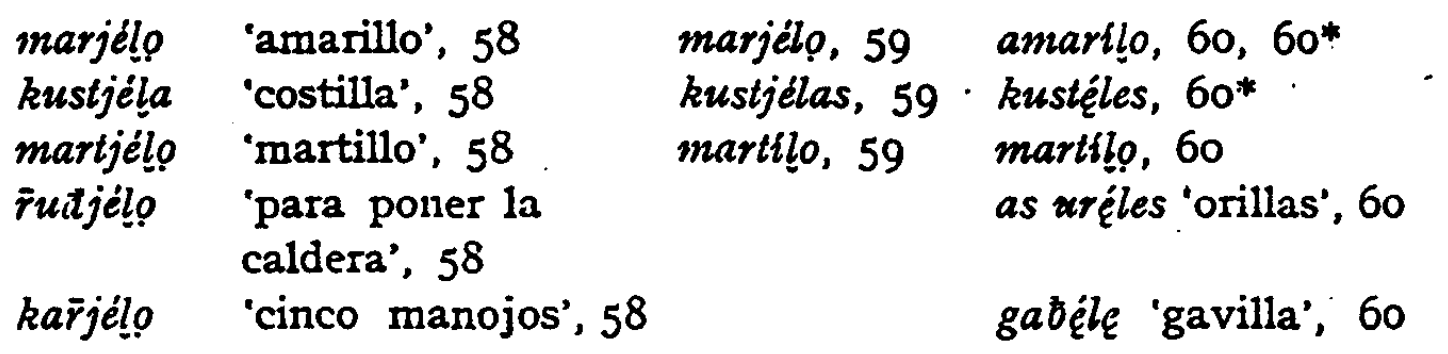

A estos ejemplos podriamos agregar muchos más.

Corresponde -jćlo, 58, al tipo ampliamente difundido en tierras leonesas; marjello, 50, 54, 55; urjélas, 5I; truitjellas 'Truchillas', 52, nombre de lugar; kućjêlo, 55; kastrjélo 54, topónimo; la bein jela, 54, topónimo, etcétera ${ }^{3}$.

-élo es la forma típicamente gallega: maręlọ, martélo, kustęles, 62; gabéla, 63, etc.

$\mathrm{Y}$ entre estos dos tipos (-jélo-élo) se intercala -jélo de Pombriego (59), una especie de compromiso, en el cual se combinan claramente la tendencia de diptongación vocálica a la leonesa y la simplificación del grupo consonántico - $l l$ - a la manera gallega. No carece de interés el que exactamente el misnio cruce pueda observarse en otra zona rayana

1 Cp. sobre la Cabrera Baja datos en TrDoRoLeon, I, 92, 124 inueite, I16, nueite Sigüeya; 92, I16, nueite, I.omba, pero en la pág. 124 otros ejemplos con $\tilde{n}$; 124 ñ-en Silván (pero noite); Trabazos, $\tilde{n}$-, pero nueite; Ilamas, ñoite; usin duda la palatalización es realmente extraña a Pombriegon, donde se registra noche frente a nuestra transcripción nweịte 59 .

2 $n$ - prevalece en el Alto León (más hacia el Norte), TrDoRoLeon, I, 3I, 34, exceptuando el grupo Sur, mapa III, Urdiales-Igüeña-Tremor, donde se usa $\tilde{n}$-.

Cp. sobre $\tilde{n}$ - $/ n$ - en dialectos leoneses, particularmente Sanabria S. Ciprian § 44; Mezcla § 22; Zauora Vicente. Dial. esp., 96-97.

Sobre castellanismos con $n$ - cp. el capitulo dedicado al mapa 'noche'.

3 Cp. sobre la difusión de -iello en dialectos leoneses el resumen de ZaMORA VICENTE. Dial. esp., 79-80; sobre Sanabria S. Cipridn, 23. 
del dominio leonés, en la zona meridional de Sanabria donde el tipo

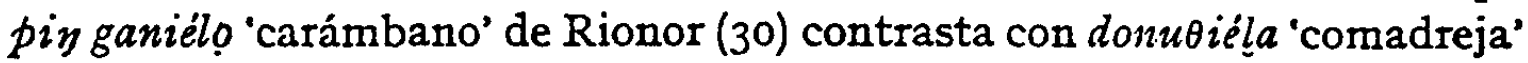
de Santa Cruz (29) y trapéla, topónimo de Hermisende (23) ${ }^{1}$.

Son de interés secundario las adaptaciones más o menos frecuentes al sufijo castellano -illo.

No encontramos en ningún otro sector de nuestro mapa 'molino' una tan marcada diferenciación de formas (todas ellas derivadas de esa misma palabra), diferenciación que se manifiesta, además, dentro de límites sumamente estrechos, como en el extremo NO del Río Cabrera. Interesa, además, que el cruce de formas - pues de tal fenómeno se trata también en el caso presente- no se ha verificado - como en casos anteriores- en Pombriego (59), sino en la zona gallega colindante. Así encontramos el tipo molíno, ampliamente difundido por la parte leonesa de Sanabria y todo el Sur de León, no sólo en Benuza (58), sino también en Pombriego $(59)^{2}$ y desde alli la designación evidentemente castellana irradió a la zona gallega ${ }^{3}$, donde aparece en la formación híbrida moling ${ }^{4}$ y la otra molingo, en la cual ya se anuncia la forma netamente gallega mọino, port. moinho, propia también de todo cl Oeste de Sanabria, de habla gallega 5.

Volveremos al problema de la $-\underline{l}$ - leonesa, fase evolutiva que consideramos como autóctona, al tratar ciertos aspectos muy similares observados en la frontera leonesa-gallega de la Cabrera Baja y en el llamado Alto León. Conviene, sin embargo, delimitar, primero, geográficamente la variante $-y$-; en cierto modo rival, no menos potente en la lucha multisecular con el patriarca fonético $-\underline{l}$-.

L a $-y-1$ e on as a va claramente separada de su predecesor $(-l-)$

1 Mezcla § 8.

2 In el mismo lugar lous vecinos (TrDoRoLeon, I, 94).

3 Observamos una infiltración parecida en Rionor, Trús os Montes molino. mulino, molineiro (LETTE DE V. Opúsculos, IV, 768) = molino, pero moére en Rionor de Castilla (30), mó́re también en 28, 29, 30; molino (30) en contraposición a pađri no, andorinla 'golondrina', a freišinna 'topon.' en el mismo lugar (Mezcla, 140).

4 Anotado por nosotros en Castroquilame.(60) asi como en TrDoRoLeon, I, 99 mulin, donde la misma forma es registrada también en lugares vecinos (12-15 del Interrogatorio) de la Cabrera gallega.

Corresponden a las formas citadas de 60: $u$ mequ padrin $60,60^{*}$, por otra parte sobrino, paürino (!).

- Cp. sobre la Cabrera gallega, TrDoRoLeon, I, 99. 
-cp. n. 6 p. $27 \mathrm{I}$ - por una línea casi vertical que en Sanabria puede ser trazada entre los lugares 6.. 9, 8.. 39, 42, 47, 48, 49.. 3I (oréxas), 33 (uriyékras), 36 (uréyas) y que se prolonga hasta la zona meridional donde Rionor auréyas (30) se separa netamente de urẹ́las-aurélas de la zona occidental colindante $\left(28,29\right.$, etc.) ${ }^{1}$. Pero lo que llama, sobre todo, la atención es -aparte de cierta uniformidad a pesar de castellanismos dispersos - la extensión geográfica del fenómeno $-y$ - comprobado hasta el margen más extremo oriental de nuestras encuestas 2.

De Rionor de Castilla (30) la etapa $-y$ - se ha propagado también a los dialectos colindantes de Trás os Montes, donde encontramos oureya 'oreja', ósveia 'oveja', coneyo 'conejo', abeia 'abeja' en Río de Onor ${ }^{3}$; abéiya, cuneio, muyer, fuóia, en Guadramil 4; speyo, paya en los dos 5.

Ira asimilación de $-y$ - a la vocal palatal $i$ observada en Rionor (30) se encuentra esporádicamente también más allá de la frontera en Guadramil: us flos, as fios-fio ${ }^{6}$.

Encontramos, por fin, como última estribación de particularidades leonesas oureya, ouveya, muyer, tiyau 'tejado', etc., en la Aliste?Sayago ${ }^{8}$.

La uniformidad obscrvada en la conservación de la etapa $-y$ - en diversos sectores de Sanabria (y en parte en zouas colindantes de Trás os Montes) se manifiesta más claramente aún en la comarca contigua de la provincia de León, desde la Cabrera ${ }^{\circ}$ hasta el $R$. Eria. (vale.

1 Cp. Mezcla § 33: moya 'moja'; fueyas 'hojas'; miybr 'mejor', etc.

2 Citaremos de entre la multitud de ejemplos análogos tan sólo 'abeja(s)': abéya, 9, 36, 37; abáya, 38; 'abejón', abiyóy, 38, 39.

Cp. IEITE DE VASCONCELIOS. Opúsculos, IV, 744, 746, 765, 768, 769; DIAS-Carval,Ho. Rio de Onor 29; en el $A L P I$, I, 'abeja': abefya.

- Cp. LEITE DE V. obr. cit. IV, 776, 777; ID., Est. phil. mir. II, 54; ID., Esquisse, 200.

- Cp. LeITe DE V. Opúsculos, IV, 730, 744.

- Mezcla § 33; LEITE, obr. cit., IV, 776; con frecuencia también en Asturias y esporádicamente en León; $\mathrm{cp}$. inás abajo.

1 TrDoRoLeon, I, I34, I35, 140; II, 136, 137; ALPI, I, 'abeja': abịiya ( $y$ con superpuesto $z$ ); nosotros hablamos anotado, en I912, ubejya frente a oubdxa (Wsp. Mdt. 107 \$ 338).

- zamora vicente. Dial. esp. io7.

- Cp. nuestro mapa 'orejas'; Casado LoBato § 36; además, sobre la Cabrera (incluso el sector Sur de Ponferrada), los datos contenidos en el instructivo artículo Sobre el habla de la Cabrera Baja, a base de interrogatorios escritos, publicado en TrDoRoLeon, I 87-I 28 (con mapa geográfico), especialmente sobre nuestro problema págs. 92, 94, 96 (sector leonés), 100 (sector gallego), I24-125. Necesitan un control exacto las grafias de algunos lugares: Traḅazos, págs. 92, 96, II9 
decir en la misma extensión hacia el Este como en Sanabria); de alli 1a $-y$ - se prolonga, con mayor o menor intensidad, a numerosas otras regiones de León: a la Maragateria ${ }^{1}$, el $\mathrm{R}$. Orbigo ${ }^{2}$ en el Este, a partes del Alto León ${ }^{3}$, y más hacia el Norte, a Oseja (ya en contacto con zonas asturianas) ${ }^{4}$ y Lomba ${ }^{5}$.

Hay que exceptuar sin embargo ciertos is lotes de carácter l e o $\mathrm{n}$ és donde se ha conservado frente a zonas colindantes netamente gallegas la etapa - $l-$. Son estos los siguientes:

I. Pombriego (59) en el último sector del R. Cabrera ${ }^{6}$ -tipo urélas-, lugar cuya posición entre Benuza (58) y Castroquilame (60) caracterizamos en un capítulo especial dedicado a problemas locales.

2. A 1 to L e ón: S. Pedro de Paradela y Bárcena de la Abadía en el Valle del Río Cua (orilla occidental), donde encontramos fuella (con diptongación a la leonesa), concello, muller, etc.; al lado de fueya, muyer del pueblo (netamente leonés) vecino Faro (en la orilla oriental

(-ll-, -y-); Llamas de Cabrera, pág. 92 (ueyos, mucyo, pero fuella); Villavieja, páginas 97, I18, I19; sobre ciertas sospechas, págs. 119, I 25, también pág. 33.

Complementan los datos de nuestro mapa algunos pueblos del sector oriental de la Cabrera que figuran en TrDoRoLeon, I, 92: me mueyo 'me mojo', los ueyos 'los ojos' Trabazos, Llamas de Cabrera; I, I25: ouveya, fiya, muyer Sigüeya (entre 57 y 58$)$.

1 GARROTE, oureya, ugüeia 'oveja', oriyones del arado.

2 TrDoRoleon, I, 54: 65 vieyo, 58 menoyo, 55 muy'a 'moja', 60 muyere, 65 uveya 'oveja', cunceyo.

- TrDoRoLeon, I, 30, 33, mapa IV: por una parte en el extremo Norte (Faro: fueya, muyer) -cp. más abajo- confrontando con - $l-$, por otra parte en la zona Sur (Urdiales, Igüeña: oureya, ureiya, muyer; Andarraso, en este último lugar rivalizando con -ch-, etapa propia de la zona Centro norte).

1 Fernández GonzAlez. Oseja, 54, 318, 319 oveya, oreya, oreyeira del arado, 180 abeyas.

Cp. inás abajo sobre la irradiación de tipos asturianos.

- Morín. Lomba, 3o, 3I: grayo 'grajo', güeyo 'ojo'.

- He aqui la caracterización acertada de aquella parte del R. Cabrera que encontramos en TrDoRoLeon, I, I25: "Ell resultado de los grupos LY y KI más generalizado en los pueblos de habla leonesa es $y$, frente a los gallegos (Puente de Domingo Flórez ( $=60^{*}$ de nuestra encuesta: oréles), Voces, Paradela, Santa Lucia, Castroquilame $(=60)$, Lago de Carucedo), que tienen siempre $l l n$; la $l$ de Pombriego (59) es considerada como rasgo gallego ("como en gallegon); el caso es, sin embargo, más complicado como evidencian los uumerosos leonesismos de dicho lugar (cp. el cap. Problemas locales). «En la provincia de Ireón los limites (entre el leonés y el gallego) deben de cstar (Menḱndez Pidar. Dial. leonés) en la divisoria de los ríos Cuá y Sil» (ZaMora VICENTE. Dial. esp., 72). 
del R. Cua); pero en contraposición a -l-folla, orella, muller en Villarbón, de habla netamente gallega ${ }^{1}$. La lateral palatal se prolonga al valle igualmente gallego de Ancares (más hacia el Norte) ${ }^{2}$.

La variedad asturiana, descrita y delimitada geográficamente en una scric de estudios recientes ${ }^{3}$, merece nuestro interés no sólo por encontrarse en clla aspectos (particularmente la $-y$-) de larga tradición y comunes con amplias zonas leonesas de León y Zamora, sino también - y hasta en grado aún mayor - porque ella revela tendencias distintas que, a primera vista, parecen - por su gran difusióncomo corrientes típicas del Norte, pero que, según toda probabilidad, han irradiado de allí a zonas vecinas del dominio leonés (especialmente de la provincia de León).

Vamos a caracterizar tal diversidad por un esquema de ejemplos escogidos que recopilamos en una zona relativamente limitada del SO de Oviedo:

sóyo 'suelo del carro' distr. de Tineo = gall. sollo

áyo 'ajo', bjéya 'vicja', agriya 'aguja', kuyarina 'renacuajo', Besullo; kuyáres 'de la rueda del carro', Trones, etimológicamente $=$ 'cucharas'

aču 'ajo', bjéća 'vieja', guça 'aguja', Bao, etc., en el sector oriental del R. Ibias.

álo 'ajo' ,Villar de Cendias, en el sector occidental del $R$. Ibias

$\bar{r} e^{y} o$ 'cuerda para atar cargas', íéa 'reja', los dos derivados de REGULA: fuéa 'hoja', fia 'hija', etc., Besullo.

Evidencian estos ejemplos los aspectos siguientes:

$-y$-: sóyo, ayo; "el resultado más general en el asturiano común"

1 Cp. el articulo Limites de palalales en el Alto León compilado por varios autores a base de un Interrogatorio escrito del año x954, publicado en TrDoRoLeon, I, 23 y sigs., especialmente págs, 30, 33. En la pág. 33 de dicho artículo se hace mención también a Tremor de Arriba, lugar situado ya más hacia el Este (zona Sur del Alto León), donde se registran fuella 'hoja', ourella 'oreja', etc.; pero no parece seguro si la grafia $l l$ es exacta (cp. $i b .$, nota I).

2 D. Alonso. Ancares, .52. abéla, obéla, etc.

2 D. Catarian, RoPh, XI, 28 y sigs.; I3I y sigs. R.-Castelinano. Aspectos, 172 y sigs., con mapa 'hoja'; nuestras Notas, 28 y sigs., 30; ZAMORA Vicente. Dial. esp., 106-108.

MENENDEZ GaRCiA. El Cuarto, I, I 45 y sigs.; Sisterna (R. Ibias) $\$ \S 5$ I, 52, 57. 58; TrDoRoLeon, II, I22, I 24 (datos sueltos); Mranterz Garcia. Cruce, 366-367 (R. Ibias: $-c h-,-l l-)$. 
(R.-Castellano, Aspectos $\S 79)^{1}$, difundido en amplias zonas de León y Zamora (cp. antes); Diego Catalán, RoPh, XI, I39.

$-\hat{c}-:$ también bastante frecuente en Asturias, dominante en el $\mathrm{SO}^{2}$ de donde ha penetrado en tierras leonesas como Valle Gordo (distr. de Murias de Paredes) ${ }^{3}$, Babia-Laciana 4 y partes del Alto León-Bierzo contiguo 5 .

-l-: rasgo típicamente gallego que encontramos en el sector occidental del Valle del $R$. Ibias -en contraposición muy clara a $-\hat{c}$ - en el sector vecino del valle desde Tablado-Bao-Sisterna lasta Degaña, etc.- así como más hacia el Norte, igualmente en las inmediaciones de Galicia en el occidente de Asturias en una estrecha faja hacia el Eo. ${ }^{6}$.

Debilitación y pérdida de la consonante intervocálica; proceso asimilatorio que se manifiesta en grados diversos $\left(-^{y}\right)$ pero que, en algunas regiones asturianas, ha conducido a una caida completa (urea, uvea, biea, etc.) "; la misina tendencia ha sido observada como fenómeno esporádico en partes de León y Zamora ${ }^{8}$, con gran regularidad en Rionor de Castilla y la zona colindante de Trás os Montes ${ }^{\circ}$.

Particularidades fonéticas y morfológicas.

No terminaremos nuestros comentarios dedicados a las múltiples variantes y problemas vinculados con la evolución de oreja sin mencionar brevemente algunas particularidades que, algún día, esperamos poder tratar más detenidamente (con referencias bibliográficas).

1 Cp. también MUNTnE, 29, 33, 34: oreya, güeyu, muyer-urea; TrDoRoLeon, II, I22, I24.

2 Cp. las fuentes citadas en especial las relativas al $R$. Ibias.

- Rubio AlvaREz, Gordo., RDiTrPop. XVII, 269 y sigs.: abecha, agucha, conceicho, navacha, fuecha, mucher, muchar 'mojar', viecho.

- Guzman Alvarez. Babia-Laciana, 269: akućar 'acoger', aćenu 'ajeno' agweicia 'ovcja'.

- TrDoRoLeon, I, 30, 33, coll mapa; ogüecha 'oveja', urecha, Páramo de Sil (GARcí REY, I2I, I54), abeça ALPI, I: 'abeja', lugar 326.

- Zamora Vicente. Dial. esp., ro6; cp. sobre -l-en zonas gallegas de Sanabria y de la Cabrera, ya antes en los capitulos respectivos; - $l-$ en el Alto León.

7 Cp. Zamora Vrcente, ro7, 108; R.-Castellano. Aspectos, i33; TrDoRo. Leon, II, I 22-I 24: ouvea, ourea, urea; MrintendEZ GARCfa. El Cuarto, I, 70; MUNTHE, 34; nuestras Notas, 30 .

S. Ciprian, 83.

- Cp. una nota anterior. 
Cp. el mapa oreja(s):

I. En la zona occidental (gallega) y meridional de Sanabria observamos una tendencia marcada a cerrar la vocal tónica ante $-l-$ e $-y$-: abęlla, ọệles.

2. Fin numerosos lugares del centro $(8,4 \mathrm{I}, 44-49)$ y en Santa Cruz (29) 1 abunda una semivocal de tránsito (ourcilla), fenómeno bastante frecuente (también ante $\hat{c})^{2}$ igualmente en otras partes del leonés y de 'Trás os Montes ${ }^{3}$.

3. Al proceso disimilatorio observado en Carbajalinos (38): oráya 'oreja', abáya 'abeja' corresponden diptongos tales como

éi >ái, óu > áu en la Cabrera Alta ' y you > yau. 'yo'

4. El numeral femenino 'dos' presenta los tipos siguientes:

dos uréyas, dos uréxas, castellanismo

diies 4i dies, I, 38; dues ourrellas, ourréyas con cierta frecuencia en el interior de Sanabria, igual que dues (duss, 13, I5) orélas en la zuna gallega (I2. I4. I6. 20) y dues aunéyas, ouréyas en la Cabrera (León); todos estos ejemplos con la debilitación típica de -as tras $140 i$ tónicas ${ }^{5}$

dus auréyas Rionor, forma que corresponde a singulares tales como

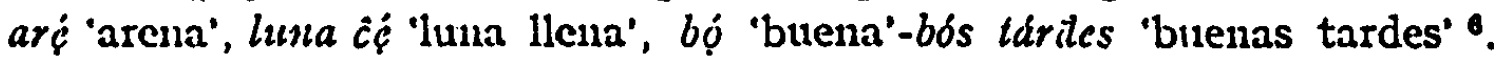

\section{LA NOCHE}

Lo que llama, a primera vista, la atención en nuestro mapa es la difusión del castellanismo nóce en amplias zonas de Sanabria (tanto en lo que se refiere a la consonante inicial, sin palatalización, como a la vocal tónica, sin diptongación, y al tratamiento del grupo consonántico $-\mathrm{KT}$-) ${ }^{2}$, contrariamente a lo que observamos en adverbios tales como 'hoy' y 'después', que allí presentan un panorama muy distinto, y en contraposición a la zona contigua de León (Cabrera) completamente arcaica en nuestro caso.

1 Mezcla \$ 20.

2 Por ejemplo en Sisterua: leiña, ouveicha.

2 Nuestros apuntes van corroborados por el $A L P I$, I: abeja: abella S. Ciprián, ab citya Rio de Onor.

- Cp. Casado I.obato, 43 y muestras observaciones en $A I L i$, IV, 276-277; R.-Castelinano. Aspectos, 200, 201; Notas 9-10.

S. Cipridn, 63-64.

- Mezcla, 133 con referencias a Trás os Montes.

7 Esperamos poder tratar todos eșos problemas bastaute complicados a base de un estudio sistemático comparativo en nuestras Muestras de un Atlas lingülstico comentado de Sanabria. 


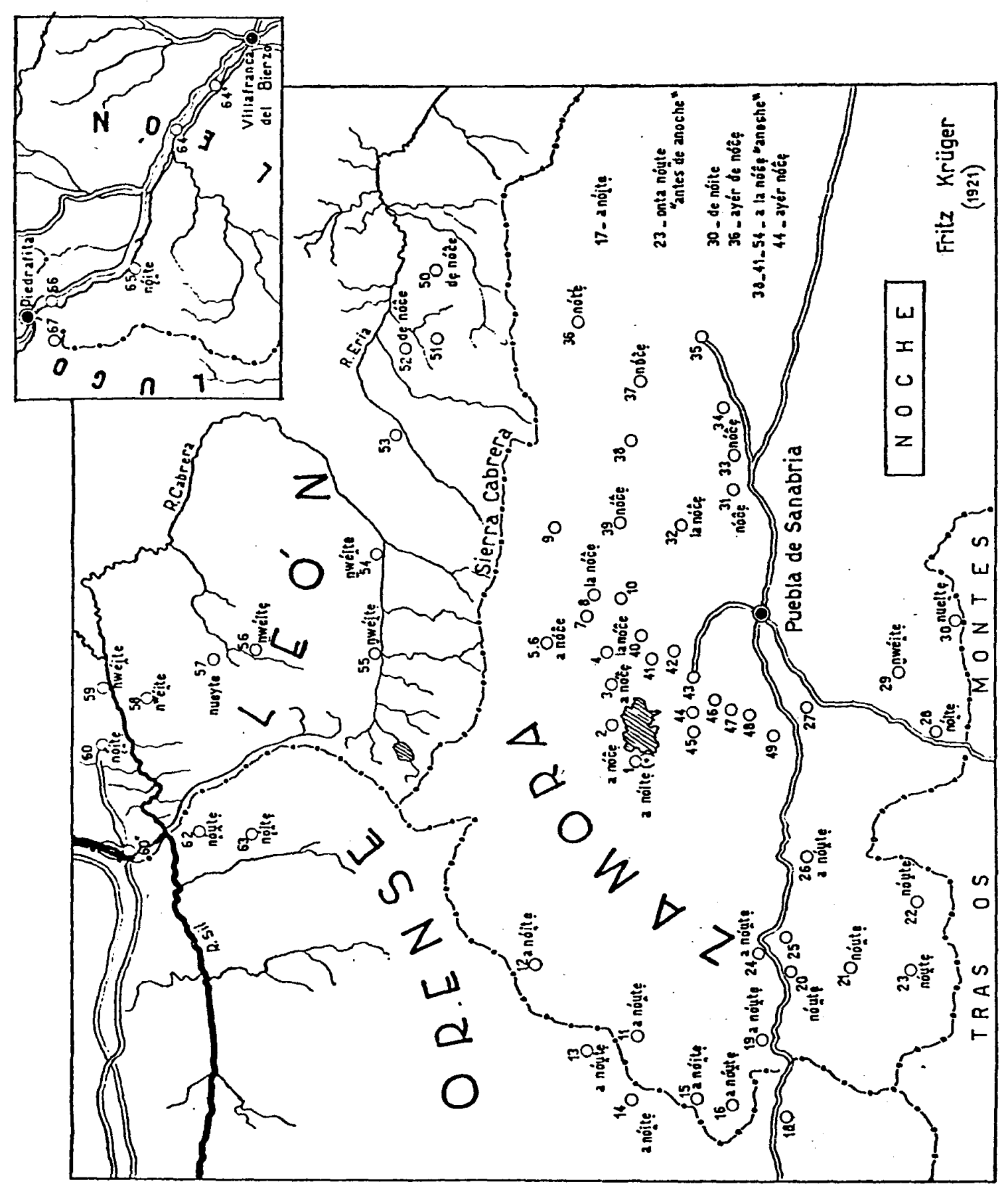


E1 carácter moderno se manifiesta en el tratamiento de la co $n$ sonante in icia 1 n- que tan sólo en el pueblo fronterizo Santa Cruz (29) -que se distingue por tendencias muy conservadoras- aparece en forma palatalizada nzwéitę; en cambio, la Cabrera casi conserva el estado de antes, igual que otras zonas de habla leonesa ${ }^{1 .}$

Presenta -entre muchos otros ejenuplos- el estado original el mapa 'nuez', en el cual aparecen en casi toda la Sanabria leonesa formas

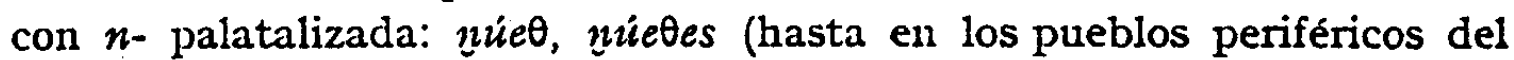

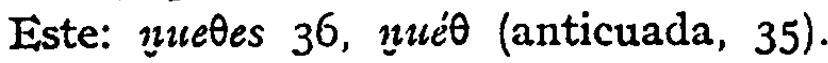

También en la dipto ng a ción típicamente leonesa de la vocal tónica ante palatal que se mantiene rigurosamente en la Cabrera (hasta Pombriego, 59) y en tantas otras regiones (siempre de habla leonesa) ${ }^{2}$ se conservan en el sector correspondiente de Sanabria en nuestro mapa pocos vestigios: nweeitite, 29, nueįte, 30; pero abundan en otros casos:

'hojas' fuelas, 4I, 42, 44, 47, 48; fuélas, 45

fwéyas, 38, al lado del castellanismo óxas como en 31, 32, $35,36,39$, pero fuyáskas

'ojos' voólus, vélos; uélọs, 29, douns ueyos 'dos ojos', 30

'después' desproóis, despwéis, despuéis; despüis, 44, 45, 48, estos últimos con simplificación del triptongo como en el caso siguiente

'hoy' groói, gwéi, guéí; gúeị; grooi đía I; gwéi đie, 32; gwui, 2, gui đie, 2, 44; gui $i, 44,45,46,47,48$ (a pocos pasos de 49, guéi).

Casi nos parece que también la forma nóite que registramos en Ribadelago (I) como anticuada y de nóite 'de noche' que en Rionor (30) aparece al lado del sustantivo nueite se derivan de esa misma forma diptongada, pero reducida -cp. la variante $n^{w}$ dite- en el uso adverbial, igual que los sustantivos no menos extraños noite, ñoite que en la Cabrera aparecen esporádicamente al lado de nueite, ñueite, tipos normales.

In cambio las formas noite, noute propias de las zonas rayanas del

1 Cp. nuestras observaciones en el capitulo Problemas locales: n-.

Sentimos no dispouer para 'noclie' de niateriales mís abundantes de los lugares 40-49; pero parece que también en ellos se usan castellanismos: a la nócé 4I, ayer nóce 44 = 'anoche'.

2 Véase también el cap. Problemas locales - noche, ojos, hoy-en el rinconcito de Benuza-Pombricgo-Castroquilame (N. de la Cabrera) con algunos datos bibliográficos provisorios. 
Oeste -Castroquilame, 60, de la Cabrera-- toda la franja occidental y, en gran parte meridional de Sanabria, II, I2 ss.-corresponden perfectamente al carácter galleguizante que las distingue ${ }^{1}$.

İRITZ KRÜGLr.

Instituto de Lingüistica de la Universidad Nacional de Cuyo (Mendoza, Argentina).

1 Cp. también despous al lado de despóns en las mismas regiones.

Sobre -oi-/-ou- cp. SCHNEIDER, VKR, XI 199 ss,; RL, XXXI, 1,75; FINK 103; D. Alonso, Ancares § 13; etc. 